\title{
Recovering the Properties of High-redshift Galaxies with Different JWST Broadband Filters
}

\author{
L. Bisigello ${ }^{1,2}$, K. I. Caputi ${ }^{1}$, L. Colina ${ }^{3}$, O. Le Fèvre ${ }^{4}$, H. U. Nørgaard-Nielsen ${ }^{5}$, P. G. Pérez-González ${ }^{6,9}$, P. van der Werf ${ }^{7}$, \\ O. Ilbert ${ }^{4}$, N. Grogin ${ }^{8}$, and A. Koekemoer ${ }^{8}$ \\ ${ }^{1}$ Kapteyn Astronomical Institute, University of Groningen, P.O. Box 800, 9700 AV, Groningen, The Netherlands \\ ${ }^{2}$ SRON Netherlands Institute for Space Research, 9747 AD, Groningen, The Netherlands; bisigello@astro.rug.nl \\ ${ }^{3}$ Centro de Astrobiología, Departamento de Astrofísica, CSIC-INTA, Cra. de Ajalvir km.4, E-28850-Torrejón de Ardoz, Madrid, Spain \\ ${ }^{4}$ Aix Marseille Université, CNRS, LAM (Laboratoire d'Astrophysique de Marseille), UMR 7326, F-13388, Marseille, France \\ ${ }^{5}$ National Space Institute (DTU Space), Technical University of Denmark, Elektrovej, DK-2800 Kgs. Lyngby, Denmark \\ ${ }^{6}$ Departamento de Astrofísica, Facultad de CC. Físicas, Universidad Complutense de Madrid, E-28040 Madrid, Spain \\ ${ }^{7}$ Sterrewacht Leiden, Leiden University, P.O. Box 9513, 2300 RA, Leiden, The Netherlands \\ ${ }^{8}$ Space Telescope Science Institute, 3700 San Martin Drive, Baltimore, MD 21218, USA \\ Received 2017 March 16; revised 2017 June 14; accepted 2017 June 15; published 2017 July 10
}

\begin{abstract}
Imaging with the James Webb Space Telescope (JWST) will allow observations of the bulk of distant galaxies at the epoch of reionization. The recovery of their properties, such as age, color excess $E(B-V)$, specific star formation rate (sSFR), and stellar mass, will mostly rely on spectral energy distribution fitting, based on the data provided by JWST's two imager cameras, namely the Near Infrared Camera (NIRCam) and the Mid Infrared Imager (MIRI). In this work we analyze the effect of choosing different combinations of NIRCam and MIRI broadband filters, from 0.6 to $7.7 \mu \mathrm{m}$, on the recovery of these galaxy properties. We performed our tests on a sample of 1542 simulated galaxies, with known input properties, at $z=7-10$. We found that, with only eight NIRCam broadbands, we can recover the galaxy age within $0.1 \mathrm{Gyr}$ and the color excess within $0.06 \mathrm{mag}$ for $70 \%$ of the galaxies. Additionally, the stellar masses and sSFR are recovered within 0.2 and 0.3 dex, respectively, at $z=7-9$. Instead, at $z=10$, no NIRCam band traces purely the $\lambda>4000 \AA$ regime and the percentage of outliers in stellar mass (sSFR) increases by $>20 \%$ ( $>90 \%$ ), in comparison to $z=9$. The MIRI F560W and F770W bands are crucial to improve the stellar mass and the sSFR estimation at $z=10$. When nebular emission lines are present, deriving correct galaxy properties is challenging at any redshift and with any band combination. In particular, the stellar mass is systematically overestimated in up to 0.3 dex on average with NIRCam data alone and including MIRI observations only marginally improves the estimation.
\end{abstract}

Key words: galaxies: high-redshift - galaxies: photometry - galaxies: fundamental parameters

\section{Introduction}

Fitting the spectral energy distribution (SED) of galaxies from broadband photometry allows derivation of a variety of galaxy properties, such as photometric redshifts, stellar masses, color excess, ages, and metallicities, with different degrees of precision, depending on the available data and used models. Particularly, SED-fitting is a powerful tool to study the properties of galaxies up to high redshifts, due to the fainter fluxes usually observable with photometry respect to spectroscopy.

The stellar mass is one of the most important quantities to derive for a galaxy, because it correlates with a large number of global properties, such as star formation rate (SFR; e.g., Brinchmann et al. 2004; Noeske et al. 2007; Rodighiero et al. 2011) and metallicity (e.g., Tremonti et al. 2004; Erb et al. 2006; Maiolino et al. 2008), and it is central in galaxy evolution. Fortunately, once the redshift is well-determined, stellar masses are one of the most robust parameters estimated from the SED-fitting (e.g., Caputi et al. 2015), while other parameters, such as age and color excess, are generally more difficult to estimate, given the degeneracy between them. However, the inclusion of nebular emission can have a large effect on the stellar masses (e.g., Stark et al. 2013; Santini et al. 2015). At low and intermediate redshifts, the emission line

\footnotetext{
${ }^{9}$ Associate Astronomer at Steward Observatory, The University of Arizona.
}

equivalent widths are relatively low for most galaxies, therefore nebular emission can be safely ignored in stellar mass calculations. However, at higher redshifts, young galaxies are more common, therefore the effect of nebular emission is expected to be more important.

In addition, stellar masses are derived by multiplying the galaxy luminosity with a mass-to-light ratio, thus the mass estimation also depends on the wavelengths of the available observations. Observations at rest-frame $\lambda>4000 \AA$, and ideally at $\lambda>1 \mu \mathrm{m}$, are necessary to derive stellar masses because the mass-to-light ratios at these wavelengths are relatively insensitive to age and color excess. Therefore, in order to derive accurate stellar masses in a wide range of redshifts, it is necessary to observe in the near-IR at low and intermediate redshifts, and in the mid-IR at high redshifts.

The James Webb Space Telescope (JWST ${ }^{10}$; Gardner et al. 2009 ) is a premier infrared space observatory for the next decade, and features a $6.5 \mathrm{~m}$ primary mirror. It has four scientific instruments on board with imaging, spectroscopic, and coronographic modes, covering a wide range of wavelengths from the visible to the mid-IR $(0.6-28 \mu \mathrm{m})$ with superb sensitivity and resolution. In particular, the two imaging cameras, namely the Near Infrared Camera (NIRCam; Rieke et al. 2005) and the Mid Infrared Instrument (MIRI; Rieke et al. 2015; Wright et al. 2015), will be used to carry out deep blank-field imaging surveys

${ }^{10}$ http://www.jwst.nasa.gov 
to study how galaxies have assembled and evolved since early cosmic times. For these surveys, SED-fitting analysis will be performed to derive galaxy properties, such as galaxy stellar mass, ages, and color excess, therefore, it is important to understand the effect of different filter combination choices on our ability to estimate these quantities.

NIRCam $^{11}$ is equipped with eight broadband filters (i.e., F070W, F090W, F115W, F150W, F200W, F277W, F356W, and $\mathrm{F} 444 \mathrm{~W})$ together with a number of medium and narrowband filters, covering the range from 0.6 to $5 \mu \mathrm{m}$. The MIRI imager $^{12}$ is complementary to NIRCam in wavelength, covering between 5 and $28 \mu \mathrm{m}$ with nine broadband filters, to observe both the redshifted stellar light at high- $z$ and the hot dust radiation at low- $z$. Only the two shortest-wavelength MIRI filters are considered in this work (i.e., F560W and F770W), because they are the most sensitive ones and therefore the ones that will be mostly used for high- $z$ galaxy studies.

Because of the complementary wavelength range covered by NIRCam and MIRI, they should ideally be both included in deep galaxy surveys, particularly for $z \geqslant 7$ where NIRCam observes the rest-frame UV/optical of the SED while MIRI covers the rest-frame optical/near-IR. However, because of the different detector technology used at near- and mid-IR wavelengths, MIRI is less sensitive than NIRCam, making observations with this instrument more time-expensive. In this work, we address this issue, analyzing the galaxy property estimation with different NIRCam and MIRI filter combinations and for different spectral templates. We treated all situations equally, without judging how common different spectral types are expected to be, in order to leave the reader decide the optimal approach for each science case.

In this work we aimed to evaluate the effect of different JWST broadband filter combinations on deriving secure galaxy properties, i.e., stellar masses, color excess, and age, for high- $z$ galaxies. We applied our test to a sample of simulated galaxies at $z=7,8,9$, and 10 derived from Bisigello et al. (2016; hereafter B16), where the photometric redshift recovery was analyzed in detail for the same combinations of JWST broadband filters.

The structure of the paper is as follows. In Section 2 we describe the analyzed sample, the obtention of the photometry in the pertinent NIRCam and MIRI bands, and the galaxy properties derivation. We present our results at different redshifts in Section 3: in particular, we analyze stellar masses, age, color excess, and specific star formation rate (sSFR). In Section 4 we summarize our main findings and conclusions. Throughout this paper, we adopt a cosmology with $H_{0}=70 \mathrm{~km} \mathrm{~s}^{-1} \mathrm{Mpc}^{-1}, \Omega_{M}=0.27, \Omega_{\Lambda}=0.73$. All magnitudes refer to the $\mathrm{AB}$ system (Oke \& Gunn 1983) and we considered a Chabrier (2003) initial mass function (IMF).

\section{Sample Selection and Test Methodology}

\subsection{Sample Construction}

Our analysis is based on a sample of 1542 simulated galaxies at $z=7-10$, presented in B16. The sample is based on simulated galaxies derived using templates from Bruzual \& Charlot (2003, BC03 hereafter); with the manual addition of the main emission lines and templates obtained with the

\footnotetext{
11 http://www.stsci.edu/jwst/instruments/nircam

12 http://www.stsci.edu/jwst/instruments/miri
}

population synthesis code Yggdrasil (Zackrisson et al. 2011). The main difference between the two types of templates is the incorporation of emission lines. The $\mathrm{BC} 03$ models do not include emission lines, so when galaxies are young and starforming, we manually added the main emission lines $(\mathrm{H} \alpha, \mathrm{H} \beta$, [O III] at $5007 \AA$ and [O II] at $3727 \AA$ ), whose rest-frame equivalent widths have been assumed to change with redshift and metallicity (see B16 for more details). In the Yggdrasil templates, nebular emission lines are automatically incorporated together with the nebular continuum emission when galaxies are young and the gas covering factor $f_{\text {cov }}>0$. For this second family of templates, rest-frame equivalent widths change with metallicity rather than redshift. In Figure 1, there is an example of a Yggdrasil SED template with $f_{\text {cov }}=1$ at the four considered redshifts $(7,8,9$, and 10$)$ to show the amount of emission lines that contaminate the JWST broadband filters analyzed in this work at each redshift.

A second difference between the two types of template is the star formation history. In particular, the $\mathrm{BCO} 3$ models have declining star formation histories, while Yggdrasil templates have step function star formation histories. It is necessary to remember this difference when analyzing the sSFR estimation for the two types of templates, as is discussed in more detail in Section 3.3. An additional BC03 SED model with increasing star formation history is analyzed in the Appendix.

For both templates we considered a large range of parameters consistent with $z=7-10$ (Table 1). We did not include galaxies with Population III stars among Yggdrasil templates, as in B16, because the stellar mass is not defined for them. All templates are normalized at $29 \mathrm{AB}$ mag at $1.5 \mu \mathrm{m}$, corresponding to the pivot wavelength of the NIRCam F150W broadband filter.

The full sample consists of 1542 galaxies: there are 417 SED models each at redshifts 7 and 8 (216 BC03 templates and 201 Yggdrasil templates) and 354 SED models each at redshifts 9 and 10 (180 BC03 templates and 174 Yggdrasil templates). The distribution of the stellar mass for all templates in this sample (corresponding to the adopted normalization) is shown in Figure 2. Differences in the two mass distributions are due to the different star formation histories used for the two types of templates and the presence of young galaxies without emission lines $\left(f_{\text {cov }}=0\right)$ among the Yggdrasil templates that are not present among $\mathrm{BC} 03$ templates. All results are valid also for lower masses, as long as the SED shapes and $\mathrm{S} / \mathrm{N}$ values are the same. Note that our sample is not intended to emulate the distribution of galaxy SEDs in a real galaxy population, but rather span the different types of SED types for the galaxies that will be observed in the high- $z$ JWSTNIRCam and MIRI blank fields, on an equal basis.

\subsection{Interpolation/Extrapolation of JWST Photometry}

All galaxies in the sample have mock observations for the 8 NIRCam broadbands, MIRI F560W and F770W broadbands from B16, derived by convolving each SED template with the NIRCam and MIRI filter transmission curves (Meyer et al. 2004; Bouchet et al. 2015; Glasse et al. 2015). We normalized each template at $29 \mathrm{AB}$ mag at $1.5 \mu \mathrm{m}$, corresponding to the NIRCam F150W filter pivot wavelength, and we scaled the fiducial stellar mass of each template accordingly to this normalization. One of the major causes of errors in the stellar mass derivation is a wrong redshift estimation. In our analysis, we wanted to study independently each parameter, therefore we 


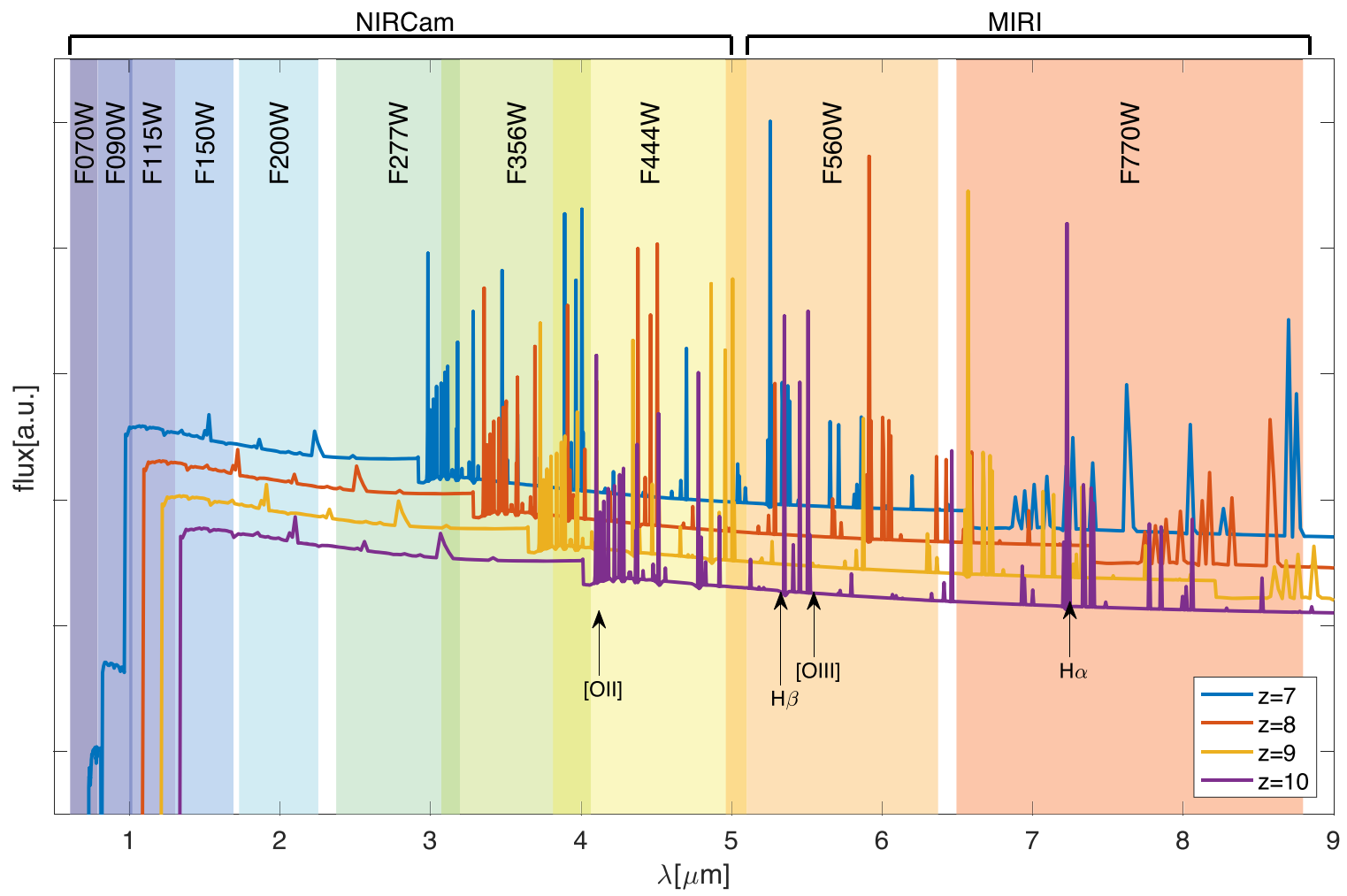

Figure 1. Four examples of SED templates from the population synthesis code Yggdrasil with $f_{\text {cov }}=1$. The colored areas specify the wavelength range of the 8 NIRCam broadband filters and MIRI F560W and F770W. This figure illustrates the amount of emission lines incorporated in these SED templates and in which JWST broadband filters they are observed at each analyzed redshift.

Table 1

Parameter Values Used to Create BC03 and Yggdrasil SED Models of the Simulated Galaxies at $z=7-10$ That Are Analyzed in This Work

\begin{tabular}{lcc}
\hline \hline Parameter & Values $(\mathrm{BC} 03)$ & Values $(\mathrm{Ygg})$ \\
\hline metallicity & $Z_{\odot}, 0.4 Z_{\odot}, 0.2 Z_{\odot}, 0.02 Z_{\odot}{ }^{\mathrm{a}}$ & $Z_{\odot}, 0.4 Z_{\odot}, 0.2 Z_{\odot}, 0.02 Z_{\odot}{ }^{\mathrm{a}}$ \\
SFH type & declining & step function \\
SFH [Gyr] & $0.01,0.1,1,10$ & $0.01,0.03,0.1$ \\
$f_{\text {cov }}$ & $\cdots$ & $0^{\mathrm{b}}, 1$ \\
$E(B-V)^{\mathrm{c}}$ & $0,0.1,0.25$ & $0,0.1,0.25$ \\
age $[\mathrm{Gyr}]$ & $0.01,0.05,0.2,0.4,0.6^{\mathrm{d}}$ & $0.01,0.05,0.2,0.4,0.6^{\mathrm{d}}$ \\
$z$ & $7,8,9,10$ & $7,8,9,10$ \\
\hline
\end{tabular}

Notes.

${ }^{\text {a }}$ For this metallicity we considered only ages $t<0.2 \mathrm{Gyr}$.

${ }^{b}$ Templates of old galaxies with no star formation ongoing do not change with the covering factor, so for these galaxies we considered only $f_{\text {cov }}=1$.

${ }^{c}$ Following the Calzetti et al. reddening law (Calzetti et al. 2000).

${ }^{\mathrm{d}}$ We considered this age only up to redshift $z=8$.

associated a signal-to-noise value of 10 with the flux at the F150W band, which corresponds to $\sim 8-9 \mathrm{hr}$ per pointing of exposure time with low background level. ${ }^{13}$ Considering these high signal-to-noise values, the photometric redshift is well determined for $>99 \%$ of the galaxies in our sample (see B16). We scaled the photometry of the other NIRCam bands considering the same integration time, but different sensitivities, according to each band. For the MIRI bands, all fluxes correspond to the SED templates normalized to $29 \mathrm{mag}$ at $1.5 \mu \mathrm{m}$. The $\mathrm{S} / \mathrm{N}$ values on the MIRI bands have been scaled assuming that a $\mathrm{S} / \mathrm{N}=10$ corresponds to $28 \mathrm{mag}$ in these

$\overline{13}$ Derived using the JWST time exposure calculator https://jwst.etc.stsci.edu/.

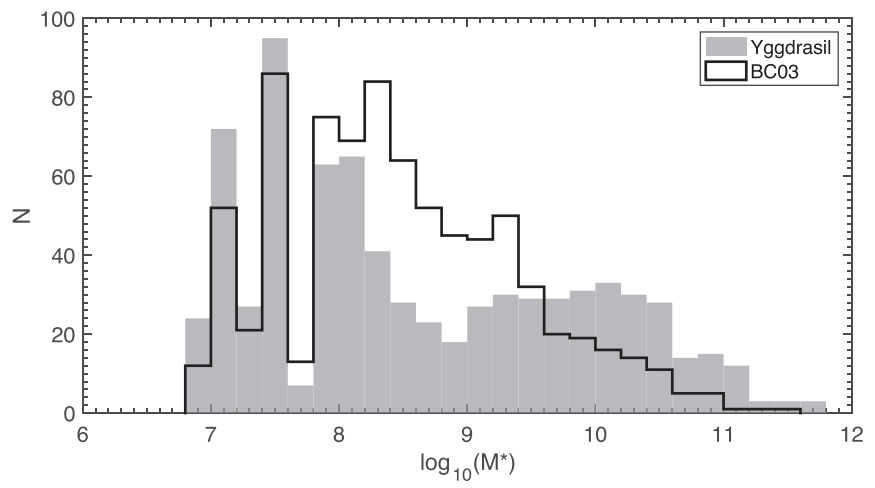

Figure 2. Input stellar masses of galaxies simulated with $\mathrm{BC} 03$ templates (black line) and Yggdrasil models (gray area). All these stellar masses correspond to SED models normalized at $29 \mathrm{AB}$ magnitude at observed $1.5 \mu \mathrm{m}$. We considered a Chabrier (2003) IMF.

bands. We considered only a signal-to-noise of 10 to limit the effects of redshift uncertainties and bad photometry. Indeed, in B16 the number of outliers in redshift (galaxies with $\left.\mid z_{\text {phot }}-z_{\text {fiducial }} /\left(1+z_{\text {fiducial }}\right)>0.15\right)$ for this $\mathrm{S} / \mathrm{N}$ value was less than $1 \%$ at all considered redshifts. Therefore, the variations in the galaxy property estimations obtained here are mainly due to degeneracies between galaxy templates and they are not expected to be solved with just a higher integration time. Differences in the $\mathrm{S} / \mathrm{N}$ value are not expected to significantly change the results of this work, as long as $\mathrm{S} / \mathrm{N} \gtrsim 10$.

A $28 \mathrm{AB}$ mag with a $\mathrm{S} / \mathrm{N}=10$ in MIRI corresponds to $\sim 196 \mathrm{hr}$ per pointing of exposure time with F560W and lowlevel backgrounds. However, as explained in more detail in Section 3.1.3, considering an $\mathrm{S} / \mathrm{N}=5$ for a $28 \mathrm{AB}$ magnitude 
Table 2

Parameter Values Used for the SED-fitting Runs of the Simulated Galaxies at $z=7-10$ That Are Analyzed in this Work

\begin{tabular}{|c|c|c|c|}
\hline Parameter & Values (BC03) & Values (Ygg) & Values (Additional BC03) \\
\hline metallicity & $Z_{\odot}, 0.4 Z_{\odot}, 0.2 Z_{\odot}, 0.02 Z_{\odot}$ & $Z_{\odot}, 0.4 Z_{\odot}, 0.2 Z_{\odot}, 0.02 Z_{\odot}$ & $Z_{\odot}, 0.4 Z_{\odot}, 0.2 Z_{\odot}, 0.02 Z_{\odot}$ \\
\hline SFH type & declining & step function & declining \\
\hline SFH [Gyr] & $0.01,0.1,1,10$ & $0.01,0.03,0.1$ & $0.01,0.1,1,10$ \\
\hline$f_{\mathrm{cov}}$ & $\cdots$ & 0,1 & $\cdots$ \\
\hline$E(B-V)^{\mathrm{a}}$ & $0,0.05,0.1, \ldots, 1$ & $0,0.05,0.1, \ldots, 1$ & $0,0.05,0.1, \ldots, 1$ \\
\hline age [Gyr] & $0.01,0.05,0.2,0.4,0.6$ & $0.01,0.05,0.2,0.4,0.6$ & $1,1.5,2.5,5$ \\
\hline$z^{\mathrm{b}}$ & $0-11$ & $0-11$ & $0-11$ \\
\hline
\end{tabular}

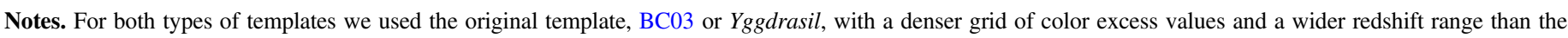
original templates used to derived the mock photometry (see Table 1). Moreover, in both cases, we also considered additional BC03 templates with old ages.

${ }^{\text {a }}$ Following the Calzetti et al. reddening law (Calzetti et al. 2000).

b This is the redshift range in the SED-fitting run.

on the MIRI bands will not create a major difference for the stellar mass estimation and it will be possible with $\sim 47 \mathrm{hr}$ per pointing of exposure time.

Each mock flux is randomized 100 times within the error bars and we considered as non-detection every flux below the $2 \sigma$ level. As a conservative approach, when running the SEDfitting code, we adopted a $3 \sigma$ upper limit, rather than $2 \sigma$, in all cases of non-detections.

More details about the JWST photometry and redshift derivation are presented in B16.

\subsection{Galaxy Properties Derivation}

We used the expected JWST photometry for our simulated galaxy sample to test how accurately it is possible to derive different properties, i.e., stellar mass, age, color excess, and sSFR. As in B16, we performed these tests with different combinations of JWST bands:

1. 8 NIRCam broadbands.

2. 8 NIRCam broadbands and 2 MIRI bands (F560W and F770W).

3. 8 NIRCam broadbands and MIRI F560W only.

4. 8 NIRCam broadbands and MIRI F770W only.

We considered these band combinations to understand in which situations NIRCam broadband filters are sufficient to properly recover galaxy properties, and in which cases adding the two shortest-wavelength MIRI filters can significantly improve the estimations. We considered only these two MIRI broadband filters because they are the most sensitive ones, thus making them the filters most likely to beused in high- $z$ galaxy surveys. As deep observations with MIRI are significantly more timeconsuming than those with NIRCam, because of different detector technology in the near and mid-IR, observation strategies may opt to use only one of the two bands. Therefore, we considered this situation by also analyzing filter combinations with only one of the two MIRI filters.

For all filter combinations, we ran the public code LePhare (Arnouts et al. 1999; Ilbert et al. 2006) to derive stellar mass, age, color excess, and sSFR, allowing for a large variety of templates. A full list of the parameter values used for the SEDfitting is presented in Table 2.

First, when running LePhare, we considered a library containing the same templates used to derive JWST photometry, i.e., the $\mathrm{BC} 03$ templates with incorporated emission lines and the Yggdrasil templates, which features a more refined grid of color excess values and $z=0-11$. However, in BC03 templates, we limited the used templates to only those corresponding to the pre-determined photometric redshift. For these templates, we added manually the main emission lines, whose rest-frame equivalent widths change with redshift and metallicity (see B16 for more details). However, we note that the use of templates that change with redshift introduces an unnecessary level of degeneracy in parameter space. Albeit the redshifts are correctly recovered in the vast majority of cases, assigning the "wrong" line equivalent widths could create more scatter in the derived stellar mass. In particular, this happens when it is not possible to determine the level of the continuum and therefore the equivalent width of the emission lines. This is the case when there is not a clean measurement of the near-IR continuum at $\lambda>4000 \AA$, without contamination by emission lines, e.g., when using only eight NIRCam broadbands at $z=7$. In this case, the level of the continuum can be determined by observing at rest-frame $\lambda>4000 \AA$ (i.e., with the F770W band at $z=7$ ), but it is necessary to increase the number of used filters to cover all possibilities in a wide range of redshifts. However, since the redshift estimation is mostly not affected by this problem and it is already possible to derive a good photometric redshift using eight NIRCam bands if the $\mathrm{S} / \mathrm{N} \geqslant 10$, we decided to first estimate the photometric redshift using all templates and then derive the stellar mass, together with age and color excess, limiting the templates to the ones corresponding to the derived redshift.

In the SED-fitting, we also included templates of older galaxies and higher extinction values than those expected at $z$ $>7$, in order to allow for degeneracies between redshift, dust, and age to be manifested. In particular, we included templates from $\mathrm{BC} 03$ with solar metallicity, ages from 0.01 to $5 \mathrm{Gyr}$, exponentially declining star formation histories with different characteristic times $\tau$ from 0.01 to $10 \mathrm{Gyr}$, and we applied color excess following the Calzetti et al. reddening law (Calzetti et al. $2000)$ with extinction values $E(B-V)=0-1$, with a step of $0.05 \mathrm{mag}$.

Each galaxy in the sample has 100 sets of photometry, obtained by randomizing fluxes within each own error bar. We derived the median of the photometric redshifts of the 100 runs of each galaxy and we recovered the parameter values of the median $z_{\text {phot }}$ solutions, despite its equivalence with the fiducial redshift, albeit the redshifts themselves are correctly recovered in most cases. So, we proceeded in two steps: first, we derived the median stellar mass among the recovered solutions, then we retrieved the parameters values of the median stellar mass solution. To summarize, we considered the parameter set of the 
template corresponding to the median photometric redshift and median stellar mass as the final output values of stellar mass, color excess, age, and sSFR of each galaxy. While we considered as fiducial values, the input values of each used an SED model.

\section{Results}

\subsection{Stellar Masses}

In this section we present our stellar mass results, derived using NIRCam broadbands alone and adding alternatively the F560W and F770W MIRI bands. All masses are derived from the normalization of each SED template, using all available bands. It is important to remember that, at $\mathrm{S} / \mathrm{N}=10$, the number of outliers in redshift $\left(\left|z_{\text {output }}-z_{\text {fiducial }}\right| /\left(1+z_{\text {fiducial }}\right)>0.15\right)$ is less than $1 \%$ for both $\mathrm{BC} 03$ and Yggdrasil templates with all band combinations (Bisigello et al. 2016). Because the considered redshifts are four fixed values $(z=7,8,9$, and 10), we showed the output mass estimation for each case separately and for each plot we quote the mean and the rms values of the $\Delta\left(\log \left(M^{*}\right)\right)=\log \left(M_{\text {output }}^{*}\right)-\log \left(M_{\text {fiducial }}^{*}\right)$ distribution, where fiducial values for the stellar mass are the input values of each template. Our results are shown in Figures 3-5 for the BC03 and Yggdrasil templates separately.

\subsubsection{Galaxies Simulated with BCO3 Templates}

For the BC03 templates, the results for the stellar mass recovery are shown in Figure 3. The outlier fractions for each JWST filter combination are listed in Table 3. Outliers are defined as galaxies with $\Delta \log (M)=\log _{10}\left(M_{\text {output }}^{*}\right)-\log _{10}\left(M_{\text {fiducial }}^{*}\right)>3 \sigma_{\log M^{*}}$, with $\sigma_{\log M^{*}}=0.04$ dex, which is the minimum $\sigma_{\log M^{*}}$ obtained with all considered filter combinations.

First, we analyzed the results considering only eight NIRCam broadband filters. The mass difference distribution is quite narrow up to $z=9$, with $\sigma_{\log M^{*}}=0.05-0.08 \mathrm{dex}$, but it becomes broad at the highest redshift, with $\sigma_{\log M^{*}}=0.29 \mathrm{dex}$. The fraction of outliers is high at all redshifts, at $z=7-9$ it is between $5.6 \%$ and $25.6 \%$, and it is even higher $(31.1 \%$ ) at $z=10$. The wings of the distribution are almost totally composed of galaxies with emission lines, which also made almost all the outliers. We will analyze galaxies with emission lines further in Section 3.1.2.

Second, we analyzed the results derived from using both MIRI bands together with the eight NIRCam broadband filters. At $z=7-8$, the incorporation of the MIRI bands is not improving the already small rms values obtained with NIRCam alone. The number of outliers when adding MIRI bands is slightly higher than when considering only NIRCam bands, however, the stellar mass offset is less then $0.15 \mathrm{dex}$ for the majority of cases, and never over 0.3 dex. Moreover, when the stellar mass estimation worsens from adding the two MIRI bands, the estimation of other parameters, such as age or color excess, generally improves (see Section 3.2.1). At $z=9$, instead, the rms value does not significantly change, but the mean does decrease as well as the number of outliers, which becomes $\sim 16 \%$.

At $z=10$, the importance of the MIRI bands becomes more evident. Indeed, the rms value becomes similar to other redshifts, decreasing from 0.29 dex with eight NIRCam bands to 0.08 dex when adding the two MIRI bands. Also, the fractions of outliers decrease from $31.1 \%$ with eight NIRCam bands to $13.3 \%$ with the two MIRI bands. This happens because few NIRCam bands cover a rest-frame wavelength redward of the $4000 \AA$ break at increasing redshifts, i.e., at $z=10$ no NIRCam broadbands purely cover $\lambda>4000 \AA$.

When the MIRI bands are considered one at a time, the rms values are generally similar to the case with both MIRI bands at all redshifts as well as the fraction of outliers, with small differences in one or the other band depending on the redshift.

Overall, when considering BC03 templates and NIRCam observations alone, the stellar mass estimation is generally good for galaxies without emission lines and for galaxies with emission lines at $z \leqslant 9$, while it becomes more difficult at $z=10$. Indeed, at $z=10$ no NIRCam band purely covers restframe $\lambda>4000 \AA$ and it is difficult to set the level of the continuum and therefore, derive the stellar mass. Adding the two MIRI bands does not significantly improve the already good mass estimation at the lowest redshifts, while it improves it substantially at $z=10$ by adding more information at $\lambda>4000 \AA$.

\subsubsection{Galaxies Simulated with Yggdrasil Templates}

Figure 4 shows the distribution of the difference between the recovered stellar masses and the fiducial ones for galaxies simulated with the Yggdrasil templates at each fixed redshift and for all JWST filter combinations. The fractions of outliers of the full sample of Yggdrasil simulated galaxies are listed in Table 4. Outliers are defined as galaxies with $\Delta \log (M)=$ $\log \left(M_{\text {output }}^{*}\right)-\log \left(M_{\text {fiducial }}^{*}\right)>3 \sigma_{\log M^{*}}$, with $\sigma_{\log M^{*}}=0.15 \operatorname{dex}$, which is the minimum $\sigma_{\log M^{*}}$ obtained with all considered filter combinations.

When considering only eight NIRCam bands, the $\Delta \log (M)$ distributions appear peaky with long tails, particularly toward positive values. The rms values are similar at $z=7$ and 8 , namely $\sigma_{\log M^{*}}=0.19$ and $0.17 \mathrm{dex}$, respectively, and they increase at higher redshifts up to $0.25 \mathrm{dex}$ at $z=10$. The fraction of outliers ranges between $4.5 \%$ at $z=8$ and $12.6 \%$ at $z=10$.

When adding both MIRI bands, the mass estimation slightly improves at all redshifts, except at $z=9$. In particular, the main improvement is in the number of outliers at $z=10$, which is reduced by more than a half. Adding the two MIRI bands at $z=9$ does not significantly change the mass estimation, except for galaxies with emission lines for which the estimation became worse. However, the estimation of other parameters, such as age and color excess, generally improves when the stellar mass does not (see Section 3.2.2). Indeed, for this particular redshift and for galaxies with emission lines, the stellar mass estimation, as well as other parameters, is also particularly difficult when including the MIRI bands. This is because emission lines are present in all bands at $\lambda>4000 \AA$, therefore it is not possible to set the level of the continuum.

When analyzing the two MIRI bands separately, we see that they have similar roles, but F560W decreases the rms and the fraction of outliers slightly more than F770W at $z>7$.

Figure 5 shows the distribution of the difference between the recovered and the fiducial stellar mass, but only for galaxies with emission lines, which are young galaxies with covering factors $f_{\text {cov }}>0$. The number of outliers is defined as galaxies with $\log \left(M_{\text {output }}^{*}\right)-\log \left(M_{\text {fiducial }}^{*}\right)>3 \sigma_{\log M^{*}}$, with $\sigma_{\log M^{*}}=0.15 \mathrm{dex}$, similar to the full Yggdrasil sample, and they are reported in Table 4 between square brackets.

When considering only eight NIRCam bands, the stellar mass is generally overestimated, with mean values between 


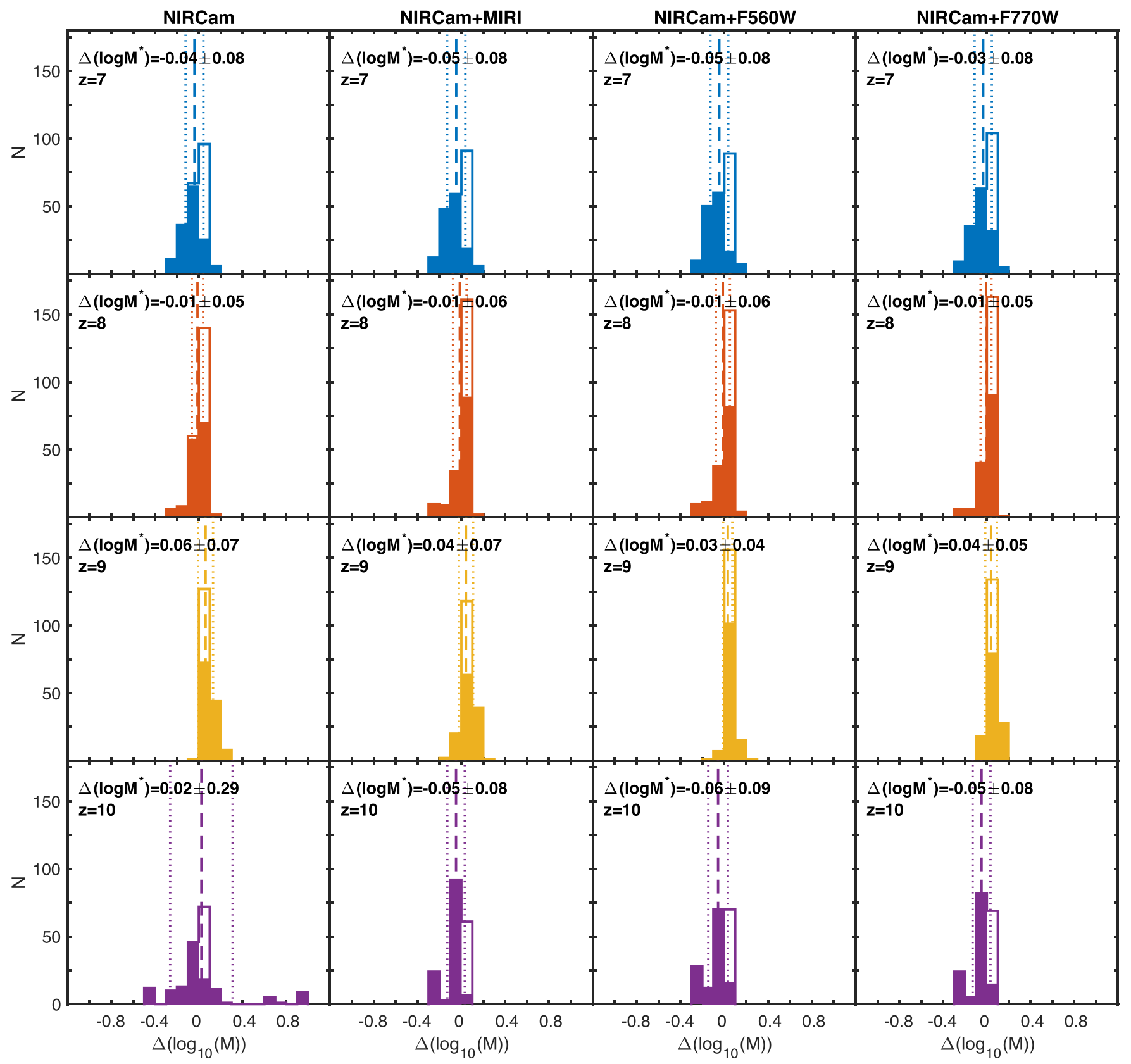

Figure 3. Differences between the derived and the fiducial stellar mass for the $\mathrm{BC} 03$ simulated galaxies at different fixed redshifts, i.e., $\Delta \log _{10}\left(M^{*}\right)=\log _{10}\left(M_{\text {output }}^{*}\right)-\log _{10}\left(M_{\text {fiducial }}^{*}\right)$. From top to bottom: redshifts $z=7,8,9$, and 10. Stellar masses in each column are obtained with different combinations of JWST bands. From left to right: eight NIRCam broadbands; eight NIRCam broadbands, MIRI F560W and MIRI F770W; eight NIRCam broadbands and MIRI F560W only; eight NIRCam broadbands and MIRI F770W only. The vertical lines indicate the mean and the $1 \sigma_{\log } M^{*}$ values, which are quoted at the top left of each panel. The colored histograms represent galaxies with emission lines, while the white ones represent galaxies without emission lines.

0.10 and 0.27 dex. The rms values are higher than the values of the general sample, ranging between 0.26 and 0.32 dex, with a minimum value at $z \leqslant 8$. Among all outliers, galaxies with emission lines are the majority, but also a small fraction of galaxies without emission lines are present, differing from the BC03 templates. Among the outliers with no emission lines, the majority (22 out of 31) are red and quenched galaxies and the remaining are blue SF galaxies with $f_{\text {cov }}=0$ that are not present among $\mathrm{BC} 03$ templates.

Adding the MIRI bands produces small improvements on the stellar mass recovery at $z=7-8$, while, as seen for the general sample, the two MIRI bands have the largest impact at $z=10$, with an rms that decreases $0.09 \mathrm{dex}$, while the mean changes from 0.25 to 0.07 dex. Similarly, when looking at the number of outliers, at $z<9$ adding the two MIRI bands creates small differences $(\sim 20 \%)$, while at $z=10$ the number of outliers is reduced to almost half. At $z=9$ the number of outliers with emission lines increases.

When considering the two MIRI bands separately, F560W improves the mass estimation slightly more than the other band, with smaller rms values at all redshifts, except at $z=9$, and lower numbers of outliers at $z>7$.

Overall, when considering Yggdrasil templates, the stellar mass estimation derived with eight NIRCam bands is generally good at $z=7-9$ but it worsens with increasing redshift. Moreover, it is particularly difficult when galaxies have nebular 


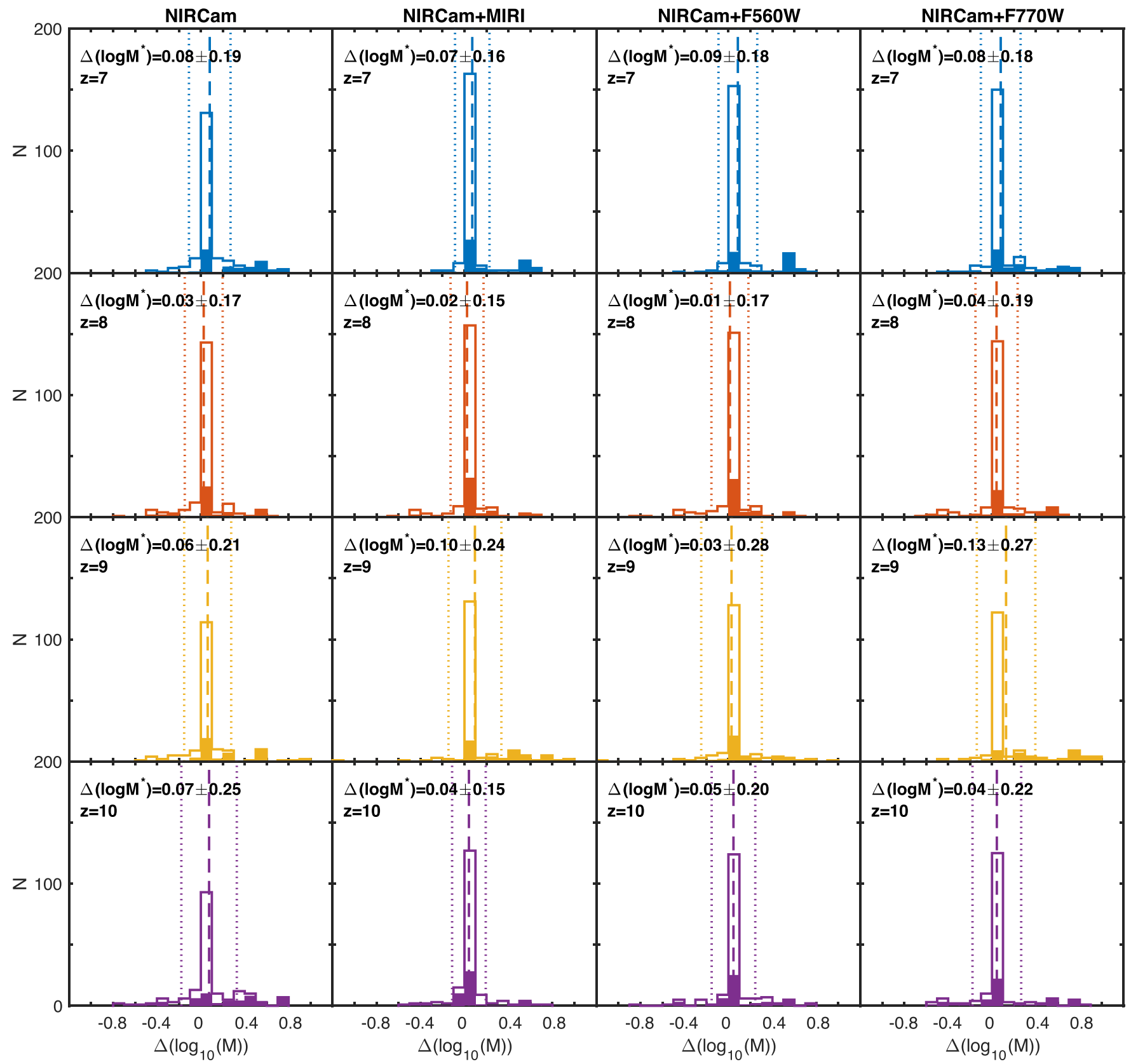

Figure 4. Differences between the derived and the fiducial stellar mass for the Yggdrasil simulated galaxies at different fixed redshifts, i.e., $\Delta \log _{10}\left(M^{*}\right)=\log _{10}\left(M_{\text {output }}^{*}\right)-\log _{10}\left(M_{\text {fiducial }}^{*}\right)$. From top to bottom: redshifts $z=7,8,9$, and 10. Stellar masses in each column are obtained with different combinations of JWST bands. From left to right: eight NIRCam broadbands; eight NIRCam broadbands, MIRI F560W and MIRI F770W; eight NIRCam broadbands and MIRI F560W only; eight NIRCam broadbands and MIRI F770W only. The vertical lines indicate the mean and the $1 \sigma_{\log } M^{*}$ values, which are quoted at the top left of each panel. The colored histograms represent galaxies with emission lines, while the white ones represent galaxies without emission lines.

emission lines. MIRI bands slightly improve the mass estimation of the general sample and particularly improves it at $z=10$, because MIRI bands cover the rest-frame $\lambda>4000 \AA$, which otherwise is purely covered by no NIRCam bands at this redshift. Moreover, adding MIRI bands improves the stellar mass recovery of galaxies with emission lines at all redshifts, except at $z=9$.

\subsubsection{Stellar Mass Recovery with $S / N=5$ with MIRI}

As explained before, the integration times for reaching an $\mathrm{S} / \mathrm{N}=10$ at $28 \mathrm{AB}$ mag with MIRI are significantly longer than those for NIRCam. However, when considering an $\mathrm{S} / \mathrm{N}=5$, instead of an $\mathrm{S} / \mathrm{N}=10$, at $28 \mathrm{AB}$ mag for the MIRI bands and leaving an $\mathrm{S} / \mathrm{N}=10$ with all NIRCam bands, the differences in the derived stellar masses are $<5 \%$ for $94 \%$ of the sample of Yggdrasil templates. This percentage varies a little with redshift, from $\sim 96 \%$ at $z=7$, to $\sim 92 \%$ at $z=10$. For the $\mathrm{BC} 03$ templates the differences in the derived stellar masses are $<5 \%$ for $79 \%$ of the sample at $z=7,87 \%$ at $z=8$, $56 \%$ at $z=9$, and $85 \%$ at $z=10$.

Reaching mag $=28$ with an $\mathrm{S} / \mathrm{N}=5$ with MIRI is indeed possible, i.e., $\sim 38(\sim 63)$ hours of exposure time with the F560W band and a low-level (medium-level) background, and the stellar mass derived is similar to the one obtained considering an $\mathrm{S} / \mathrm{N}=10$ with MIRI for the majority of cases. 


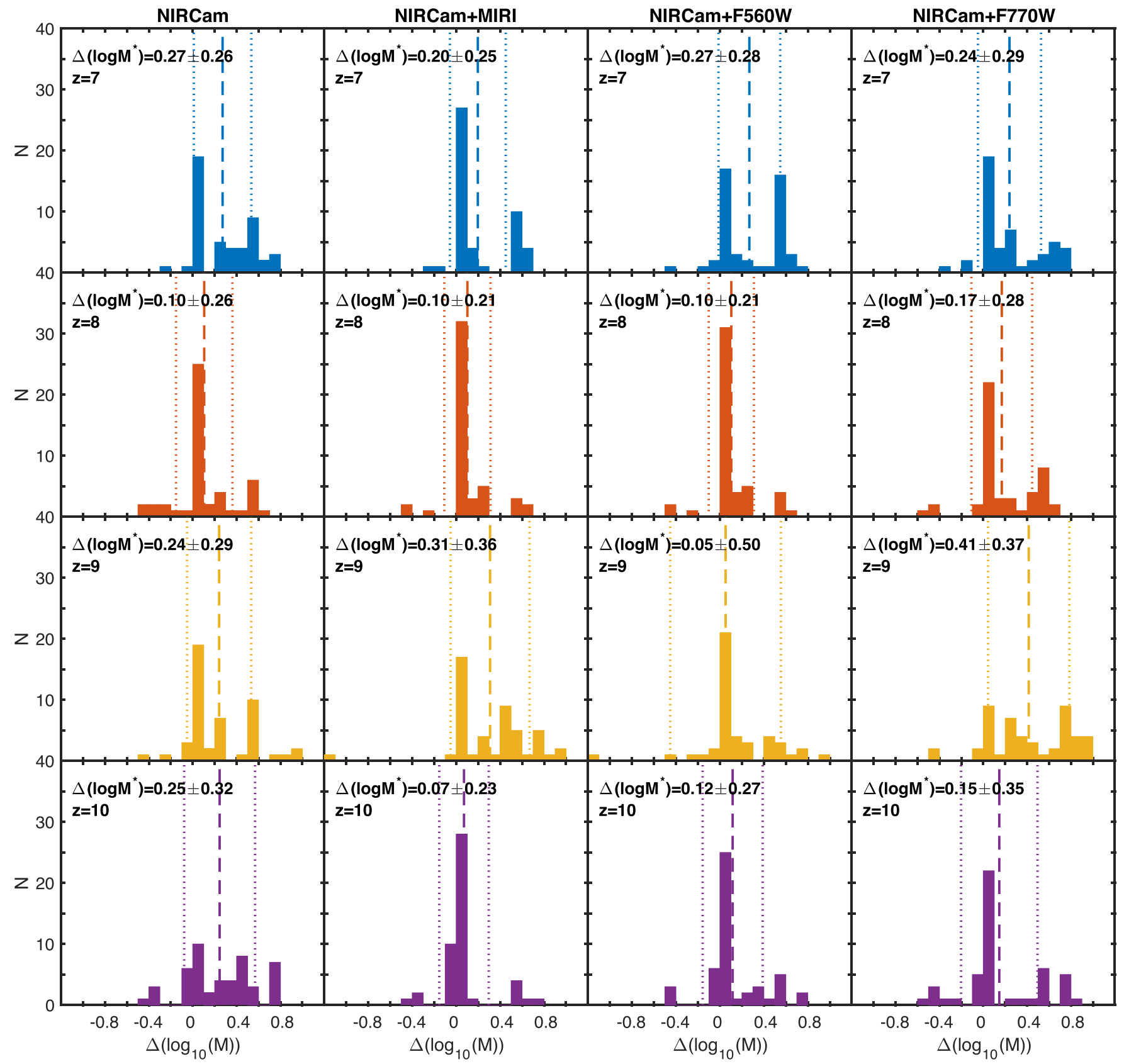

Figure 5. Zoom-in of Figure 4 showing only galaxies with emission lines.

\subsection{Age and Color Excess}

In this section we present our results of age and color excess recovery for galaxies derived both with the $\mathrm{BC} 03$ templates and with theYggdrasil models. Because of the age-dust degeneracy, we decided to analyze the two properties together. Figures 6-8 show the difference between the derived and fiducial ages compared to the difference between the derived and fiducial color excess values at each fixed redshift, for the $\mathrm{BC} 03$ and Yggdrasil templates. For each plot we quote the mean and rms values of both the age difference $\left(t_{\text {output }}-t_{\text {fiducial }}\right)$ and the color excess difference $\left(E(B-V)_{\text {output }}-E(B-V)_{\text {fiducial }}\right)$ distributions. As for the stellar masses, we present our results derived using observations with only eight NIRCam broadband filters, adding both MIRI bands and only one of the two separately.

\subsubsection{Galaxies Simulated with BCO3 Templates}

In Figure 6 we show the comparison between fiducial and output values, both for age and color excess, for the $\mathrm{BC} 03$ templates. Outliers are defined as galaxies with $\mid t_{\text {output }}-t_{\text {fiducial }}>3 \sigma_{t} \quad\left(\left|E(B-V)_{\text {output }}-E(B-V)_{\text {fiducial }}\right|>\right.$ $\left.3 \sigma_{E(B-V)}\right)$, with $\sigma_{t}=0.01 \mathrm{Gyr}\left(\sigma_{E(B-V)}=0.02 \mathrm{mag}\right)$, which is the minimum rms value for the age (color excess) obtained with all considered filter combinations.

When considering only eight NIRCam bands, the color excess is estimated within $|\Delta E(B-V)|<0.2$ mag for all objects at all redshifts, the mean of the distribution is $<0.02 \mathrm{mag}$ and the rms values are $0.02-0.06 \mathrm{mag}$, with the highest value at $z=7$. The number of outliers is high at $z=7$ and it decreases with redshift, from $25.5 \%$ to $0.0 \%$. On the 
Table 3

Number (Percentage) of Outliers in Stellar Mass among the 792 (216 Each at $z=7$ and 8, and 180 Each at $z=9$ and 10) Galaxies Simulated with BC03 Templates and Manual Addition of Emission Lines, for Different Combinations of JWST Filters and Redshifts

\begin{tabular}{|c|c|c|c|c|}
\hline Bands & $N_{\text {outlier }, z=7}$ & $N_{\text {outlier }, z}=8$ & $N_{\text {outlier }, z}=9$ & $N_{\text {outlier }, z}=10$ \\
\hline 8 NIRCam broadbands & $41[41](19.0 \%)$ & $12[12](5.6 \%)$ & $46[46](25.6 \%)$ & $56[55](31.1 \%)$ \\
\hline 8 NIRCam bands+MIRI F560W, F770W & $50[50](23.1 \%)$ & $21[21](9.7 \%)$ & $28[28](15.6 \%)$ & $24[24](13.3 \%)$ \\
\hline 8 NIRCam bands+MIRI F560W & $50[50](23.1 \%)$ & $24[24](11.1 \%)$ & $10[10](5.6 \%)$ & $34[34](18.9 \%)$ \\
\hline 8 NIRCam bands+MIRI F770W & 39 [39] $(18.1 \%)$ & $11[11](5.1 \%)$ & $14[14](7.8 \%)$ & $25[25](13.9 \%)$ \\
\hline
\end{tabular}

Note. Between square brackets is the number of galaxies with emission lines among the outliers. Outliers are defined as those values beyond $3 \sigma_{\log } M^{*}$ of the distribution $\Delta \log _{10}\left(M^{*}\right)=\log _{10}\left(M_{\text {output }}^{*}\right)-\log _{10}\left(M_{\text {fiducial }}^{*}\right)$, with $\sigma_{\log M^{*}}=0.04$ dex.

Table 4

Number (Percentage) of Outliers in Stellar Mass among the 750 (201 Each at $z=7$ and 8, and 174 Each at $z=9$ and 10) Galaxies Simulated with Yggdrasil Templates, 192 of which Are with Emission Lines, for Different Combinations of JWST Filters and Redshifts

\begin{tabular}{|c|c|c|c|c|}
\hline Bands & $N_{\text {outlier }, z=7}$ & $N_{\text {outlier }, z=8}$ & $N_{\text {outlier }, z}=9$ & $N_{\text {outlier, } z=10}$ \\
\hline 8 NIRCam broadbands & $16[15](8.0 \%)$ & $9[7](4.5 \%)$ & $16[15](9.2 \%)$ & $22[16](12.6 \%)$ \\
\hline 8 NIRCam bands+MIRI F560W, F770W & $16[14](8.0 \%)$ & $6[5](3.0 \%)$ & $21[20](12.1 \%)$ & 9 [7] $(5.2 \%)$ \\
\hline 8 NIRCam bands+MIRI F560W & $22[22](10.9 \%)$ & $9[6](4.5 \%)$ & $13[13](7.5 \%)$ & $14[10](8.0 \%)$ \\
\hline
\end{tabular}

Note. Between square brackets is the number of galaxies with emission lines among the outliers. Outliers are defined as those values beyond $3 \sigma_{\log } M^{*}$ of the distribution $\Delta \log _{10}\left(M^{*}\right)=\log _{10}\left(M_{\text {output }}^{*}\right)-\log _{10}\left(M_{\text {fiducial }}^{*}\right)$, with $\sigma_{\log _{10} M^{*}}=0.15$ dex.

other hand, the age difference distribution has rms values between 0.05 (at $z=9$ ) and $0.10($ at $z=7$ ) and the mean values are between -0.03 and $0.02 \mathrm{Gyr}$. However, long tails are present up to $|\Delta t|=0.4 \mathrm{Gyr}$ and the number of outliers ranges from a minimum of $15.0 \%$ at $z=9$ and a maximum of $36.1 \%$ at $z=7$. In general, the age and the color excess rms values decrease with the redshift also because the age-dust degeneracy is reduced due to the decreasing age of the universe.

When adding both MIRI bands, the rms of the color excess difference does not change significantly, but the number of outliers decreases at $z \leqslant 8$. On the other hand, the age rms slightly decreases at all redshifts, but the number of outliers decreases only at $z=7$ and 10 . Moreover, at $z>7$ the age is never overestimated, while the color excess is never underestimated.

The two MIRI bands play similar roles when considered separately and neither of them improve the age or the color excess estimation particularly more than the other one at all redshifts.

Similarly to the mass estimation, most of the outliers, both in age and color excess, are galaxies with emission lines.

To conclude, with only NIRCam bands, both the color excess and age estimation distribution present long tails and extreme outliers. The two MIRI bands improve the age estimations, mainly reducing the rms, while they mainly reduce the number of outliers in the color excess estimation.

\subsubsection{Galaxies Simulated with Yggdrasil Templates}

In Figure 7 we show the comparison between fiducial and output values, both for age and color excess, for Yggdrasil templates, for each redshift and different band combinations. Outliers are defined as galaxies with $\left|t_{\text {output }}-t_{\text {fiducial }}\right|<3 \sigma_{t}$ $\left(\left|E(B-V)_{\text {output }}-E(B-V)_{\text {fiducial }}\right|<3 \sigma_{E(B-V)}\right)$, with $\sigma_{t}=$ $0.01 \mathrm{Gyr}\left(\sigma_{E(B-V)}=0.01 \mathrm{mag}\right)$ which is the minimum rms value obtained for the age (color excess) with all considered filter combinations.
When considering only eight NIRCam bands, the color excess estimation is similar at all redshifts, with $\sigma_{E(B-V)}=0.04$ mag. The number of outliers is also similar at all redshifts $(\sim 20 \%-30 \%)$, with a minimum value at $z=8$. The age difference distribution has $\sigma_{t}=0.02-0.08 \mathrm{Gyr}$, with a maximum value at $z=8$, while the number of outliers is between $12.9 \%$ and the $25.3 \%$, increasing with redshift.

When including the two MIRI bands, both the age and the color excess estimation are generally improved. In particular, for the color excess, the rms value greatly decreases at $z=7$ anddoes not change significantly at the other redshifts, while the number of outliers decreases at all redshifts and in particular at $z=7$ and 10, where it is reduced to less than half. At $z=9$, the rms slightly increases, because of a single galaxy for which the color excess is highly overestimated (0.04 mag); otherwise, without this outlier, it would remain unchanged with respect to the value derive with only NIRCam broadbands. When considering the age estimation, the rms values became $0.01 \mathrm{Gyr}$ at all redshifts, decreasing the values derived with only eight NIRCam bands, except at $z=7$ where it remains 0.2 Gyr. The number of outliers decreases from $13 \%$ to $25 \%$, when considering only NIRCam bands, to $\sim 10 \%$ with both MIRI bands. Moreover, there are no galaxies with $|\Delta t|>0.1$ Gyr at $z>8$.

The two MIRI bands improve age and color excess similarly, but the F560W decreases the rms of both the age difference and the color excess difference a bit more than the other band.

In Figure 8, we show the comparison between fiducial and output values, both for age and color excess, but only for galaxies with emission lines, which are young star-forming galaxies with covering factors $f_{\text {cov }}>0$.

The color excess estimation for galaxies with emission lines does not present significant differences from the full Yggdrasil sample, both when considering only eight NIRCam bands and when adding the two MIRI bands. The same thing happens generally to the age estimation. As for the stellar mass, not all 


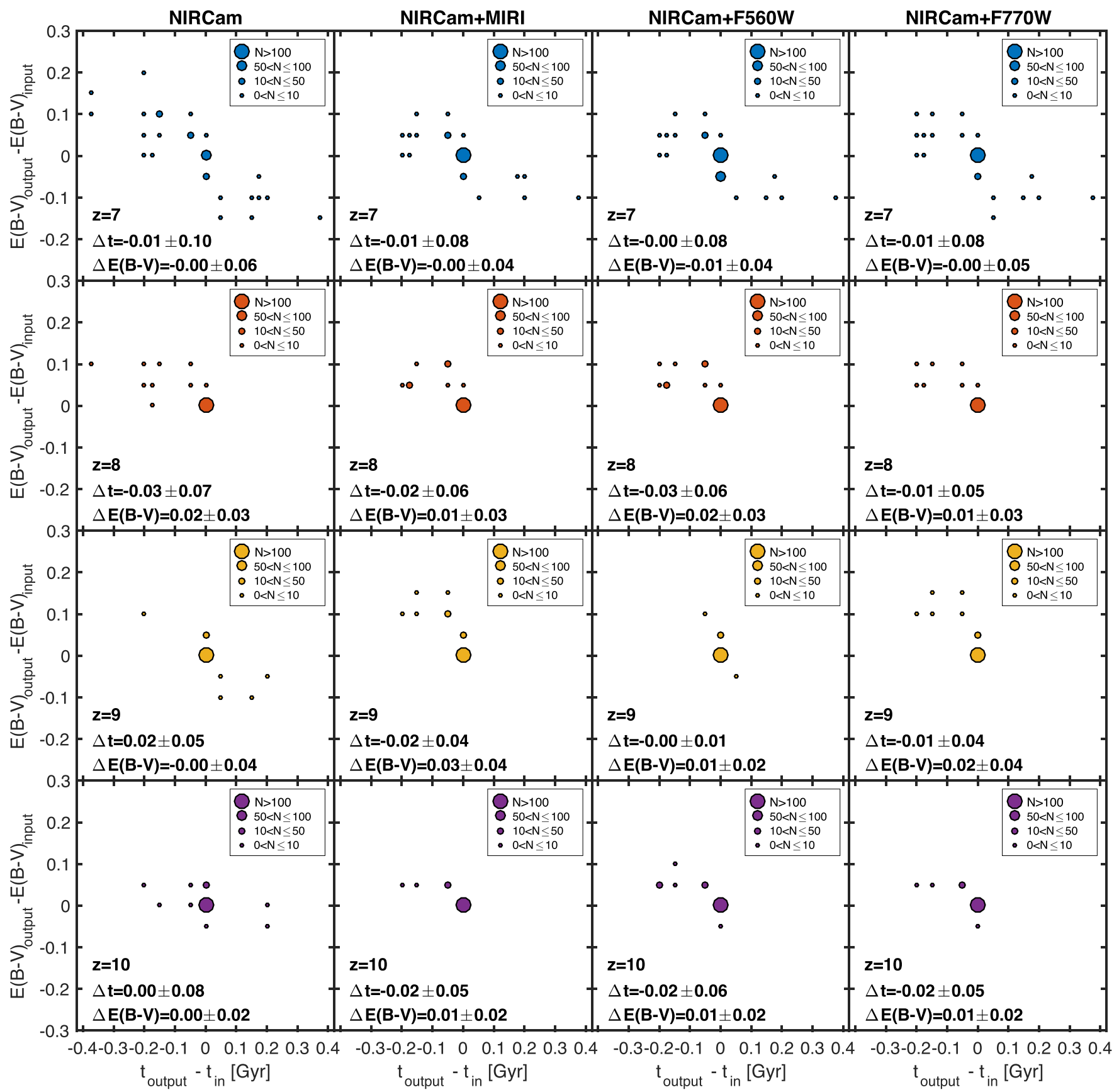

Figure 6. Difference between the derived age and the fiducial one, $t_{\text {output }}-t_{\text {fiducial }}$, against the difference between the derived color excess and the fiducial one, $E(B-V)_{\text {output }}-E(B-V)_{\text {fiducial }}$, for galaxies simulated with the $\mathrm{BC} 03$ templates at different fixed redshifts. From top to bottom: redshifts $z=7,8,9$, and 10 . Ages in each column are obtained with different combinations of bands. From left to right: eight NIRCam broadbands; eight NIRCam broadbands, MIRI F560W and MIRI F770W; eight NIRCam broadbands and MIRI F560W only; eight NIRCam broadbands and MIRI F770W only. Galaxies are divided into bins of 25 Myr in age and $0.025 \mathrm{mag}$ in color excess and the size of each circle represents the number of galaxies inside each bin. The size of the dots increases with the number of galaxies inside the respective bin. Mean and rms values of the age and the color excess estimation are shown at the bottom left of each panel.

outliers in age and color excess are galaxies with emission lines.

To conclude, when considering only the eight NIRCam bands, both the age and the color excess difference distributions present long tails. Adding the two MIRI bands improves both the age and the color excess estimation, slightly decreasing the rms values, but mainly reducing the number of outliers.

\subsection{Specific Star Formation Rates}

In this section we present our results for the sSFR of star-forming galaxies derived both with the $\mathrm{BC} 03$ templates and with the Yggdrasil models. As is explained later in more detail, in the BC03 and Yggdrasil SED templates, SFR $\propto M^{*}$. So, to decouple stellar mass effects, we considered the sSFR instead of the SFR. Results are shown in Figures 9-10 at four fixed redshift values and for different band 


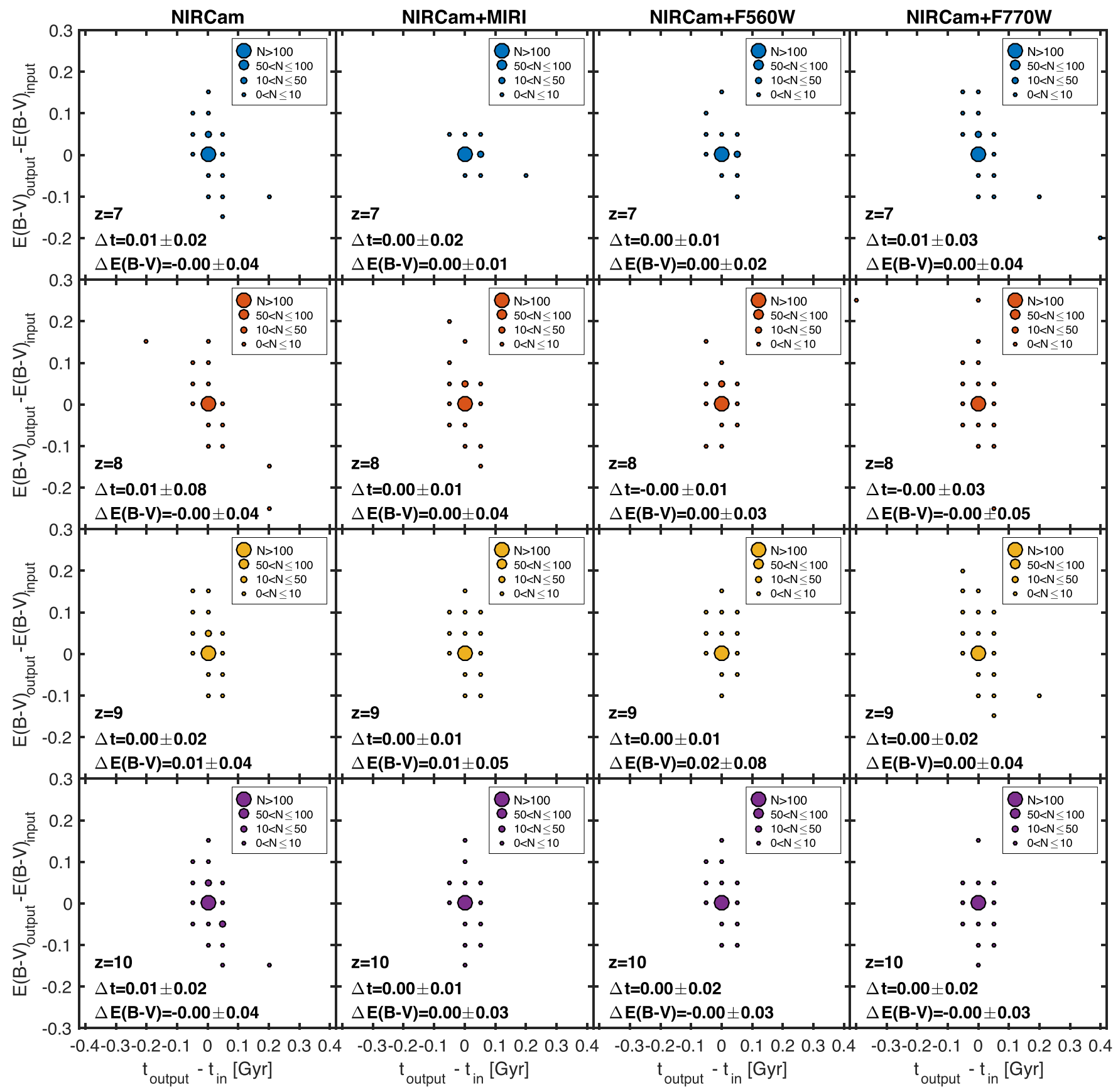

Figure 7. Difference between the derived age and the fiducial one, $t_{\text {output }}-t_{\text {fiducial }}$, against the difference between the derived color excess and the fiducial one, $E(B-V)_{\text {output }}-E(B-V)_{\text {fiducial }}$, for galaxies simulated with the Yggdrasil templates at different fixed redshifts. From top to bottom: redshifts $z=7,8,9$, and 10 . Ages in each column are obtained with different combinations of bands. From left to right: eight NIRCam broadbands; eight NIRCam broadbands, MIRI F560W and MIRI F770W; eight NIRCam broadbands and MIRI F560W only; eight NIRCam broadbands and MIRI F770W only. Galaxies are divided into bins of 25 Myr in age and 0.025 mag in color excess and the size of each circle represents the number of galaxies inside each bin. The size of the dots increases with the number of galaxies inside the respective bin. Mean and rms values of the age and the color excess estimation are shown at the bottom left of each panel.

combinations. In each case we quote the mean and the rms values of $\Delta\left(\log _{10}(\mathrm{sSFR})=\log _{10}\left(\mathrm{sSFR}_{\text {output }}\right)-\log _{10}\left(\mathrm{sSFR}_{\text {input }}\right)\right.$.

\subsubsection{Galaxies Simulated with BC03 Templates}

For BC03 templates, which are characterized by exponentially declining star formation histories, the SFR is derived as:

$$
\mathrm{SFR}_{\text {out }}=\frac{e^{\frac{-t_{\text {out }}}{\text { Tout }}}}{\tau_{\text {out }}} \frac{M_{\text {out }}}{M_{0}\left(t_{\text {out }}, \tau_{\text {out }}\right)},
$$

where $t_{\text {out }}, \tau_{\text {out }}$, and $M_{\text {out }}$ are the derived values for the age, the characteristic timescale of the declining star formation history, and the stellar mass. $M_{0}$ is the mass of the original BC03 template that is used to normalize each template to one solar mass before the SED-fitting and it depends on the age and $\tau$ value of each template. The sSFRs of the $\mathrm{BC} 03$ templates depend both on the derived age and star formation history, and not on the derived stellar mass, because $\mathrm{sSFR}_{\text {out }}=$ $\mathrm{SFR}_{\text {out }} / M_{\text {out }}$. 
The comparison between the original and derived sSFRs is shown in Figure 9 for all star-forming galaxies, which correspond to all galaxies with emission lines. Outliers are defined as galaxies with $\log _{10}\left(\mathrm{sSFR}_{\text {output }}\right)-\log _{10}\left(\mathrm{sSFR}_{\text {fiducial }}\right)>3 \sigma_{\log (\mathrm{sSFR})}$, with $\sigma_{\log (\mathrm{SSFR})}=0.14$ dex, which is the minimum $\sigma_{\log (\mathrm{sSFR})}$ obtained with all considered filter combinations. All galaxies simulated with BC03 templates are correctly identified as star-forming at each redshift and for all band combinations.

When considering only eight NIRCam bands, the sSFR difference distribution is broad at $z=7$ and 10 with $\sigma_{\log (\mathrm{SSFR})}=0.38 \mathrm{dex}$ and $0.49 \mathrm{dex}$, respectively, and it is narrower at the two intermediate redshifts with $\sigma_{\log (\mathrm{SSFR})} \sim 0.20$ dex. Similarly, the number of outliers is high at $z=7$ and 10 , $\sim 34 \%$ and $29 \%$, respectively, and it decreases at intermediate redshifts to $\sim 15 \%-16 \%$. The mode is approximately null at $z \geqslant 8$. On the other hand, the mean is around 0 at $z=7$ and 10, but the distribution is more asymmetric at the other two redshifts with $\langle\log (\mathrm{sSFR})\rangle=0.12 \mathrm{dex}$ at $z=8$ and -0.12 dex at $z=9$. The SSFR derivation depends on the age estimation, and this is particularly evident at $z=7-9$. In particular, at $z=7$ the age difference distribution is broad, therefore the SSFR difference distribution has a similar behavior. At $z=8$, the age is always correct or underestimated, so, following Equation (2), the sSFR difference is only positive or null, while the opposite happens at $z=9$. At $z=10$, the age estimation is better than it is at $z=7$, because the decreasing age of the universe limits the age uncertainties; however, no NIRCam band purely covers restframe $\lambda>4000 \AA$ and estimation of the star formation history, which is therefore the characteristic time $\tau$, becomes challenging, as well as the sSFR estimation.

When adding the two MIRI bands, the sSFR estimation improves at $z=7$ and at $z=10$, where the $\sigma_{\log (\mathrm{sSFR})}$ decreases by 0.08 and $0.27 \mathrm{dex}$, respectively. Similarly, the number of outliers decreases by $\sim 40 \%$ at these two redshifts. At $z=7$, the MIRI bands improve the age estimation and therefore the sSFR difference distribution is narrower than what is seen with only the NIRCam bands. On the other hand, at $z=10$, the MIRI bands improve the $\tau$ estimations covering $\lambda>4000 \AA$, which are otherwise purely observed by no NIRCam band. At $z=8$, both the rms and the number of outliers are similar to the values derived with only NIRCam bands, but the number of galaxies with $\Delta \log _{10}$ (sSFR) $\sim 0$ slightly increases. At $z=9$, both the rms and the number of outliers slightly increase. The age is generally properly estimated at this redshift, but the $\tau$ estimation is very difficult because emission lines are present in all bands at $\lambda>4000 \AA$.

When considering the two MIRI bands separately, they have similar influence in the sSFR estimation. However, the F560W band reduces the outlier number slightly more at $z=7-9$ than does the other MIRI band, but neither of them decreases the rms values at all redshifts more than the other.

Overall, with only the eight NIRCam broadbands, the sSFR estimation is good at $z=8$ and 9. However, it becomes more difficult at $z=7$, because the age estimation is more uncertain, and at $z=10$, because no NIRCam band purely covers restframe $\lambda>4000 \AA$, therefore the $\tau$ estimation is challenging. Adding the two MIRI bands improves the sSFR estimation at $z=7$ and 10 , but not at intermediate redshifts where it is already good.

\subsubsection{Galaxies Simulated with Yggdrasil Templates}

For Yggdrasil templates, which are characterized by a step function star formation history, the SFR is derived as:

$$
\mathrm{SFR}_{\mathrm{out}}=\frac{\mathrm{SFR}_{\mathrm{constant}} M_{\mathrm{out}}}{M_{0}\left(t_{\mathrm{out}}, \mathrm{SFH}\right)}
$$

where $\mathrm{SFR}_{\text {constant }}$ has different values depending on the durations of the star formation $(0.01,0.03$, or $0.1 \mathrm{Gyr})$, and $M_{\text {out }}$ is the derived stellar mass, while $M_{0}$ is the original mass of the template that it used to normalize each template to $1 M_{\odot}$ before the SED-fitting and it does depend on the SFH and the age. Therefore, for the Yggdrasil templates, the sSFR, defined as $\mathrm{sSFR}_{\text {out }}=\mathrm{SFR}_{\text {out }} / M_{\text {out }}$, depends mainly on the $\mathrm{SFH}$, and only marginally on the age.

The comparison between the original and derived sSFRs is shown in Figure 10 for all star-forming galaxies. Among the Yggdrasil templates, there are two types of star-forming galaxies, those with and without emission lines, depending on their covering factor. The sSFR depends mainly on the SFH, which has three discrete values, and as a consequence, the sSFR difference does not show a smooth distribution, but three separated peaks, a central one and two secondary ones, at all redshifts and with all band combinations. Therefore, we defined outliers as galaxies in the two secondary peaks, i.e., $\log _{10}\left(\mathrm{sSFR}_{\text {output }}\right)-\log _{10}\left(\mathrm{sSFR}_{\text {fiducial }}\right)>0.2$ dex. Some starforming galaxies simulated with Yggdrasil templates are wrongly identified as quiescent, i.e., instantaneous $\mathrm{SFR}=0$, and their fractions are listed in Table 5 for different redshifts and filter combinations.

First, we start our analysis considering only the eight NIRCam bands. Between $3 \%$ and $6 \%$ of star-forming galaxies are wrongly identified as quiescent at all redshifts and they are mainly galaxies with $f_{\text {cov }}=1$. This is because the presence of numerous emission lines inside all bands can be misidentified as a higher continuum with no emission lines, i.e., a red quiescent galaxy. The rms value of the sSFR difference distribution is around 0.3 dex up to $z=9$ and then it increases at $z=10-0.40$ dex. Similarly, the number of outliers is between $20 \%$ and $25 \%$ at $z=7-9$ and it increases to $40 \%$ at the highest redshift. Outliers are mainly galaxies with $f_{\text {cov }}=1$, and indeed the presence of numerous emission lines inside all bands redward of the $4000 \AA$ break complicates the identification of the continuum and therefore the estimation of the SFH. Similarly, at $z=10$ no NIRCam band purely covers rest-frame $\lambda>4000 \AA$, therefore a proper estimation of the SFH history is difficult.

When adding the two MIRI bands, less than $4 \%$ of starforming galaxies are wrongly identify as quiescent, improving the identification with only NIRCam bands at all redshifts, except at $z=9$. The rms values are $\sim 0.26-28$ at all redshifts, creating a small improvement up to $z=9$ and a more significant one at $z=10$. Similarly, the number of outliers is between $15 \%$ and $20 \%$, slightly improving at all redshifts, especially at $z=10$. As noted earlier, the MIRI bands at $z=10$ can trace $\lambda>4000 \AA$, which improves the SFH estimation and consequentially the sSFR derivation.

When considering each MIRI band separately, for F560W the fraction of star-forming galaxies wrongly identified as quiescent is smaller than that for F770W. On the other hand, the two bands similarly improve the rms values and the number of outliers, with no clear preference. 


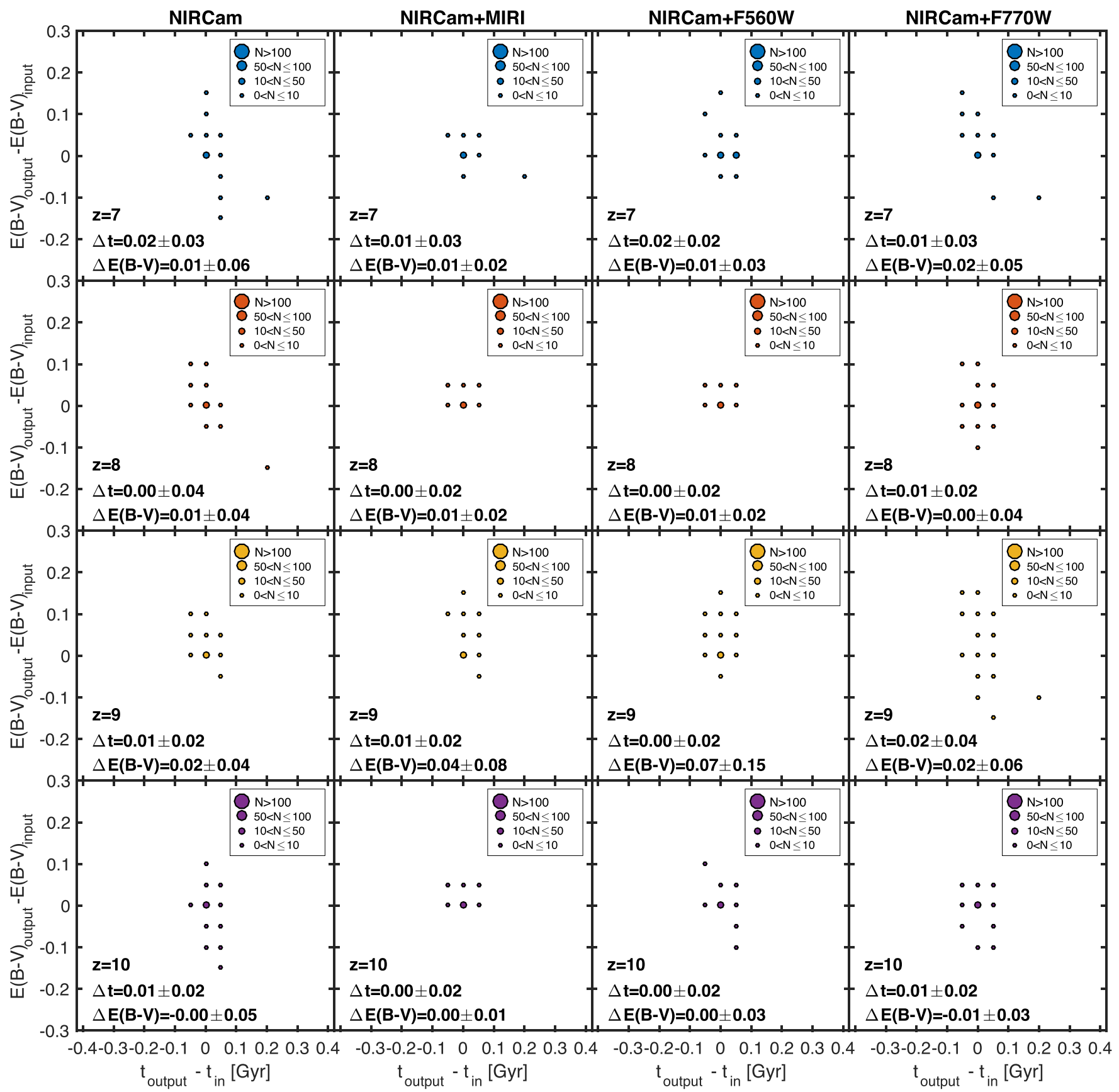

Figure 8. Difference between the derived age and the fiducial one, $t_{\text {output }}-t_{\text {fiducial }}$, against the difference between the derived color excess and the fiducial one, $E(B-V)_{\text {output }}-E(B-V)_{\text {fiducial }}$, for galaxies simulated with the Yggdrasil templates and emission lines at different fixed redshifts. From top to bottom: redshifts $z=7,8,9$, and 10. Ages in each column are obtained with different combinations of bands. From left to right: eight NIRCam broadbands; eight NIRCam broadbands, MIRI F560W and MIRI F770W; eight NIRCam broadbands and MIRI F560W only; eight NIRCam broadbands and MIRI F770W only. Galaxies are divided into bins of $25 \mathrm{Myr}$ in age and $0.025 \mathrm{mag}$ in color excess and the size of each circle represents the number of galaxies inside each bin. The size of the dots increases with the number of galaxies inside the respective bin. Mean and rms values of the age and the color excess estimation are shown at the bottom left of each panel.

Overall, with only observations with the NIRCam bands it is possible to correctly estimate the SFH for $\sim 80 \%$ of the galaxies up to $z=9$, but the estimation is more difficult at the highest redshift. Adding the two MIRI bands helps to improve the SFH estimation at all redshifts, especially at $z=10$.

\section{Summary and Conclusions}

In this work we have tested the impact that having data in different JWST filter combinations has on derivations of stellar mass, age, color excess, and sSFR for a sample of simulated galaxies at $z=7-10$. In particular, we considered the eight NIRCam broadband filters and the two MIRI filters at the shortest wavelengths (F560W and F770W), which are the most sensitive ones among all MIRI filters and those which will be preferred for high- $z$ galaxy surveys.

Our sample consists of 1542 simulated galaxies from B16, derived from $\mathrm{BC} 03$ templates with the manual addition of emission lines for star-forming galaxies (which are the ones with ages lower than the characteristic time $\tau$ of the star 


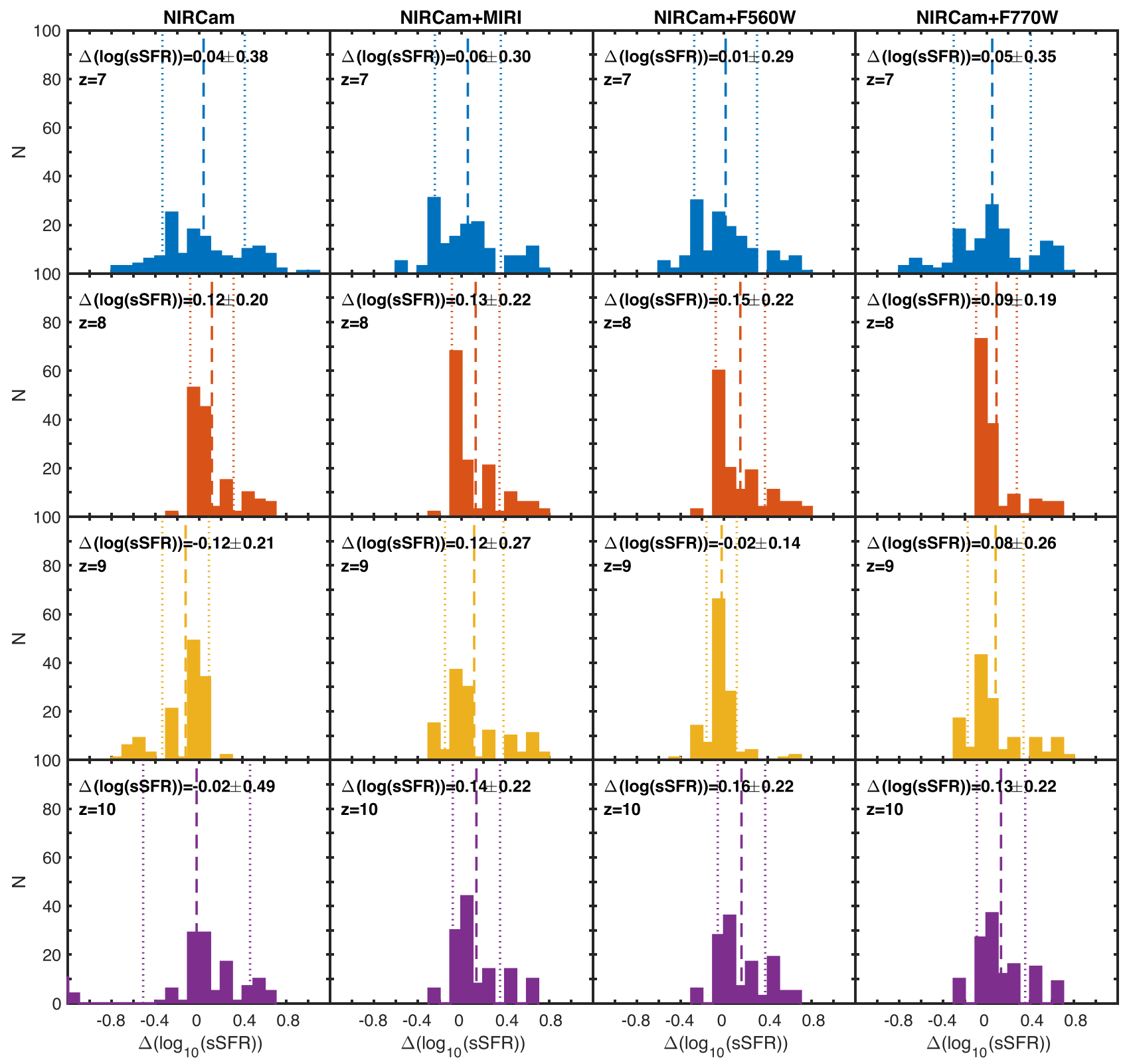

Figure 9. Differences between the derived and the fiducial sSFR for the BC03 simulated, star-forming galaxies at different fixed redshifts, i.e., $\Delta \log _{10}(\mathrm{sSFR})=\log _{10}\left(\mathrm{sSFR}_{\text {output }}\right)-\log _{10}\left(\mathrm{sSFR}_{\text {fiducial }}\right)$. From top to bottom: redshifts $z=7,8,9$, and 10 . sSFRs in each column are obtained with different combinations of JWST bands. From left to right: eight NIRCam broadbands; eight NIRCam broadbands, MIRI F560W and MIRI F770W; eight NIRCam broadbands and MIRI F560W only; eight NIRCam broadbands and MIRI F770W only. The vertical lines indicate the mean and the $1 \sigma_{\log (\mathrm{sSFR})}$ values, which are quoted at the top left of each panel.

formation history) and Yggdrasil models. All galaxies in our sample have good photometric redshift estimations, as derived in B16, therefore uncertainties on the photometric redshifts are not a significant source of error on the galaxy property estimations performed here. The sample contained the typical SED types of the galaxies that will be observed in the JWST NIRCam and MIRI high- $z$ blank surveys, in order to test potential problems on deriving galaxy properties, on an equal basis. Therefore, the used sample does not try to emulate the real distribution of galaxies at high redshift.

Our main results are:

1. For galaxies with conventional SED types (i.e., BC03 templates), with the presence of at most some prominent emission lines, stellar masses can be well recovered with NIRCam broadband data alone up to $z=9$, provided that these data have sufficiently high $\mathrm{S} / \mathrm{N}$ values $(>10)$. At $z=10$, no NIRCam filter purely covers rest-frame $\lambda>4000 \AA$, therefore the stellar mass estimation is more difficult, resulting in $31.1 \%$ of $3 \sigma_{\log } M^{*}$ outliers. For the same reason, the MIRI bands have an important role in improving the mass estimation at this redshift, reducing the number of outliers to $\sim 13 \%$.

2. When templates with rest-frame equivalent widths that evolve with redshift are considered, it is preferable to derive stellar masses in two steps, in order to avoid the code to use a template with rest-frame equivalent widths of different redshifts. First, one should derive the 


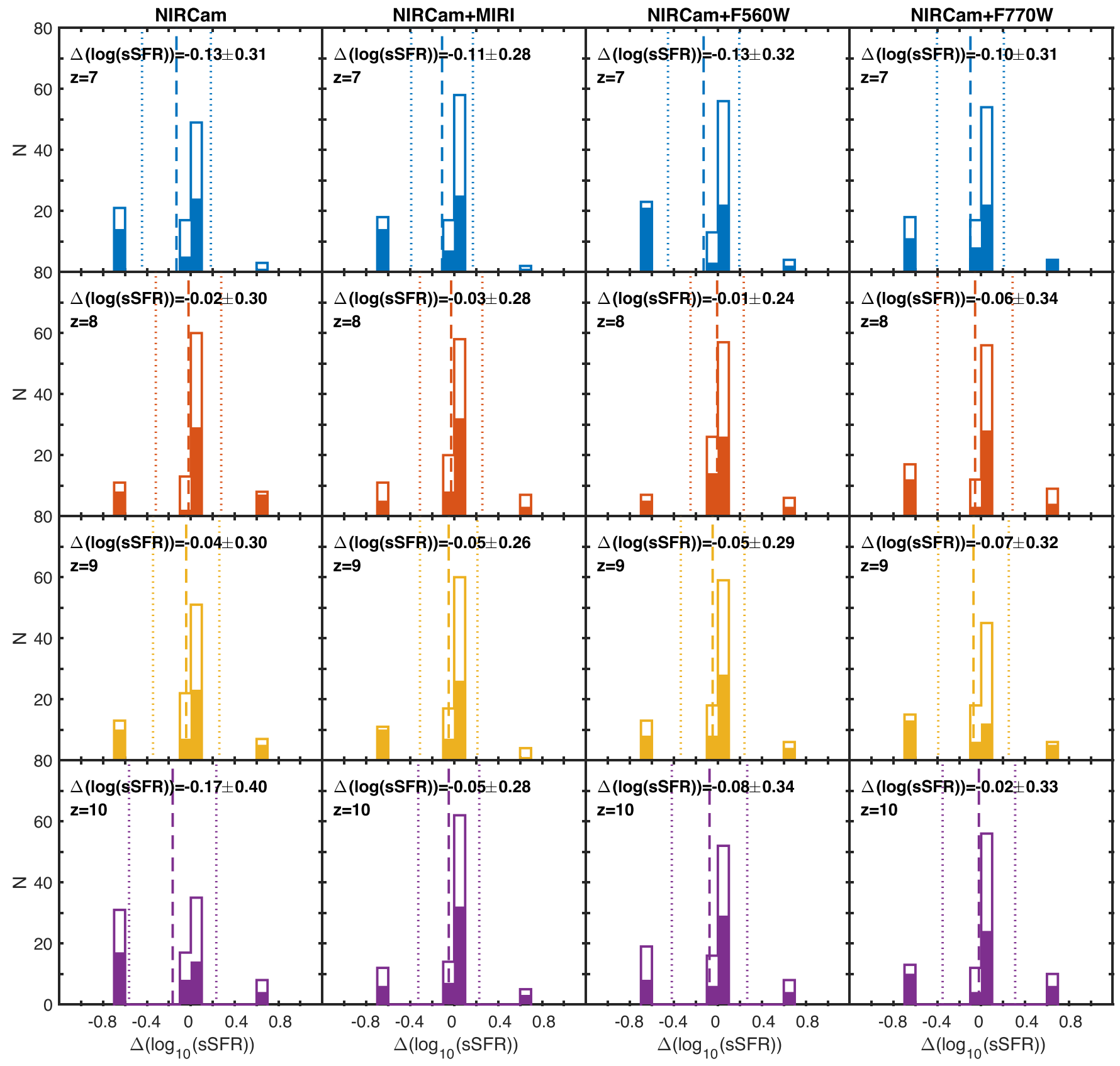

Figure 10. Differences between the derived and the fiducial sSFRs for the Yggdrasil simulated, star-forming galaxies at different fixed redshifts, i.e., $\Delta \log _{10}(\mathrm{sSFR})=\log _{10}\left(\mathrm{sSFR}_{\text {output }}\right)-\log _{10}\left(\mathrm{sSFR}_{\text {fiducial }}\right)$. From top to bottom: redshifts $z=7,8,9$ and 10 . sSFRs in each column are obtained with different combinations of JWST bands. From left to right: 8 NIRCam broadbands; 8 NIRCam broadbands, MIRI F560W and MIRI F770W; 8 NIRCam broadbands and MIRI F560W only; 8 NIRCam broadbands and MIRI F770W only. The vertical lines indicate the mean and the $1 \sigma_{\log (\mathrm{sSFR})}$ values, which are quoted at the top-left of each panel. The colored histograms represent galaxies with $f_{\text {cov }}=1$, while the white ones represent galaxies with $f_{\text {cov }}=0$.

Table 5

Number (Percentage) of Galaxies among the 384, 96 at Each Redshift, Star-forming Galaxies Simulated with the Yggdrasil Templates That Are Wrongly Identified as Quiescent, for Different Combinations of JWST Filters and Redshifts

\begin{tabular}{|c|c|c|c|c|}
\hline Bands & $N_{\text {outlier }, z}=7$ & $N_{\text {outlier }, z}=8$ & $N_{\text {outlier }, z}=9$ & $N_{\text {outlier }, z}=10$ \\
\hline 8 NIRCam broadbands & $6[2](6.5 \%)$ & $4[2](4.2 \%)$ & $3[0](3.1 \%)$ & $5[0](5.2 \%)$ \\
\hline 8 NIRCam bands+MIRI F560W, F770W & $1[0](1.0 \%)$ & $0[0](0.0 \%)$ & $4[0](4.2 \%)$ & $3[3](3.1 \%)$ \\
\hline 8 NIRCam bands+MIRI F560W & $0[0](0.0 \%)$ & $0[0](0.0 \%)$ & $4[0](4.2 \%)$ & $3[0](3.1 \%)$ \\
\hline 8 NIRCam bands+MIRI F770W & $3[0](3.1 \%)$ & $2[1](2.1 \%)$ & $12[0](12.5 \%)$ & $5[1](5.2 \%)$ \\
\hline
\end{tabular}

Note. Templates are equally divided between $f_{\text {cov }}=0$ and 1 and the number of misidentified galaxies with $f_{\text {cov }}=0$ is shown in squared brackets. 


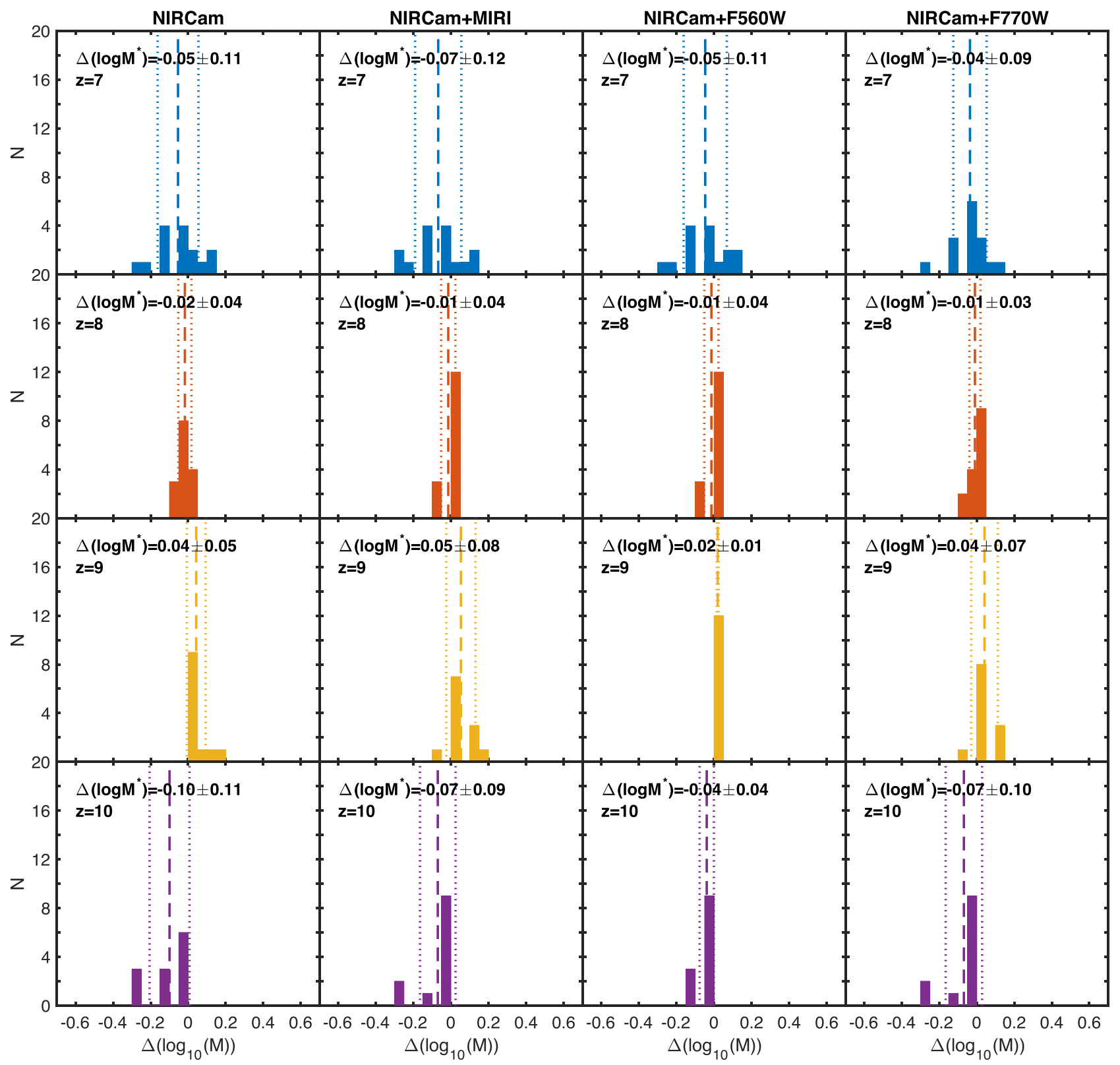

Figure 11. Differences between the derived and the fiducial stellar mass for the BC03 simulated galaxies with delayed SFHs at different fixed redshifts, i.e., $\Delta \log _{10}\left(M^{*}\right)=\log _{10}\left(M_{\text {output }}^{*}\right)-\log _{10}\left(M_{\text {fiducial }}^{*}\right)$. From top to bottom: redshifts $z=7,8,9$, and 10. Stellar masses in each column are obtained with different combinations of JWST bands. From left to right: eight NIRCam broadbands; eight NIRCam broadbands, MIRI F560W and MIRI F770W; eight NIRCam broadbands and MIRI F560W only; eight NIRCam broadbands and MIRI F770W only. The vertical lines indicate the mean and the $1 \sigma_{\log } M^{*}$ values, which are quoted at the top left of each panel.

photometric redshift, which is virtually not affected by this problem, and after that, derive the stellar mass at the fixed obtained redshift.

3. For galaxies with nebular continuum and emission, the stellar mass is generally overestimated when considering only NIRCam broadband observations. The incorporation of MIRI bands is fundamental for a proper stellar mass recovery, except at $z=9$, where the stellar mass recovery remains challenging. The reasons are that, first, the number of NIRCam bands at $\lambda>4000 \AA$ decreases with redshift, and second, emission lines contaminate these bands. In particular, at $z=9$ emission lines are inside all NIRCam and MIRI bands at $\lambda>4000 \AA$ and it is not possible to set the level of the continuum, making the mass estimation challenging.
4. The NIRCam broadband filters are sufficient to recover galaxy age for $>64 \%$ of the sample within $0.03 \mathrm{Gyr}$, and to recover the color excess for $>70 \%$ of the sample within 0.06 mag. However, extreme outliers are present at all redshifts, particularly at $z<9$. Indeed, at higher redshifts, thanks to the decreasing age of the universe, the age-dust degeneracy is reduced.

5. The two MIRI bands improve both the age estimation and the color excess estimation, slightly decreasing the rms values, but mainly reducing the number of outliers.

6. With observations in the 8 NIRCam broadband filters, the sSFR estimation is challenging at $z=10$, where no NIRCam band purely covers $\lambda>4000 \AA$, so adding MIRI observations improves the sSFR derivation. At lower redshifts, NIRCam alone is generally sufficient to 
recover the sSFR for $\sim 70 \%-80 \%$ of star-forming galaxies within 0.4 dex.

The results presented in this paper are a useful reference for the design of deep imaging surveys with JWST and to understand in which situation the time investment on MIRI observations is necessary to recover galaxy properties, i.e., stellar masses at the highest redshift. Indeed, for the majority of $7 \leqslant z \leqslant 9$ galaxies, it is possible to estimate the stellar mass, the sSFR, the age, and the color excess at all redshifts just using eight NIRCam bands. However, adding the MIRI bands, both F560W and F770W, helps improve the estimation of these quantities, and it is essential to recover the stellar mass and the sSFR of galaxies at $z=10$. On the other hand, determining the stellar masses of young star-forming galaxies with emission lines $\left(f_{\text {cov }}>0\right)$ remains challenging at any redshift. Overall, it will be necessary to observe with both cameras in order to perform an accurate study of galaxy evolution and mass assembly since early cosmic times.

L.B. and K.I.C. acknowledge the support of the Nederlandse Onderzoekschool voor de Astronomie (NOVA). K.I.C. also acknowledges funding from the European Research Council through the award of the Consolidator Grant ID 681627BUILDUP. O.L.F. acknowledges funding from the European Research Council Advanced Grant ID 268107-EARLY. P.G. P.-G. acknowledges support from the Spanish Government MINECO Grants AYA2012-31277 and AYA2015-70815ERC. L.C. acknowledges support from the Spanish Government MINECO Grants AAYA2012-32295.

\section{Appendix Increasing Star Formation Histories}

Recent simulations of the high-redshift universe predict that galaxies at $z>6$ have SFRs that on average increase with time (Finlatoret al. 2011; Jaacks et al. 2012; Zackrisson et al. 2017). Following these predictions, we tested the stellar mass derivation for a set of templates with a single increasing SFH. In particular, we considered a $\mathrm{BC} 03$ template with a delayed star formation history with a $\tau$ value of $1 \mathrm{Gyr}$, for which the SFR increases up to $1 \mathrm{Gyr}$ and then exponentially declines. We considered solar metallicity and the same extinction and age values used for the other BC03 templates. In particular, all ages used are below $1 \mathrm{Gyr}$, so all templates analyzed in this appendix have rising SFHs. We manually added emission lines as done for the other BC03 templates. We derived mock photometry for the JWST bands and the output parameters, fixing the redshift, as is described in this paper for the other BC03 SED templates.

The distribution of the difference between the derived stellar mass and the fiducial ones is shown in Figure 11. With only eight NIRCam bands the stellar mass estimation is good at $z=8-9$, while the rms is 0.11 at the other redshifts. Adding the two MIRI bands mainly improves the stellar mass estimation at $z=10$, which is otherwise generally underestimated with only eight NIRCam bands. The F560W generally improves the stellar mass recovery with respect to F770W.

This simple test does not take into account possible degeneracies among delayed star formation histories with different $\tau$ values or different metallicities. However, as for the other BC03 templates with emission lines, the stellar masses are generally correctly derived with eight NIRCam bands at $z=7-9$ and the inclusion of MIRI bands is necessary to improve the recovery of the stellar mass at $z=10$.

\section{References}

Arnouts, S., Cristiani, S., Moscardini, L., et al. 1999, MNRAS, 310, 540 Bisigello, L., Caputi, K. I., Colina, L., et al. 2016, ApJS, 227, 19 Bouchet, P., García-Marín, M., Lagage, P.-O., et al. 2015, PASP, 127, 612 Brinchmann, J., Charlot, S., White, S. D. M., et al. 2004, MNRAS, 351, 1151 Bruzual, G., \& Charlot, S. 2003, MNRAS, 344, 1000

Calzetti, D., Armus, L., Bohlin, R. C., et al. 2000, ApJ, 533, 682 Caputi, K. I., Ilbert, O., Laigle, C., et al. 2015, ApJ, 810, 73 Chabrier, G. 2003, PASP, 115, 763

Erb, D. K., Shapley, A. E., Pettini, M., et al. 2006, ApJ, 644, 813 Finlator, K., Oppenheimer, B. D., \& Davé, R. 2011, MNRAS, 410, 1703 Gardner, J. P., Mather, J. C., Clampin, M., et al. 2009, ASSP, 10, 1 Glasse, A., Rieke, G. H., Bauwens, E., et al. 2015, PASP, 127, 686 Ilbert, O., Arnouts, S., McCracken, H. J., et al. 2006, A\&A, 457, 841 Jaacks, J., Nagamine, K., \& Choi, J. H. 2012, MNRAS, 427, 403 Maiolino, R., Nagao, T., Grazian, A., et al. 2008, A\&A, 488, 463 Meyer, M. R., Rieke, M., Eisenstein, D., et al. 2004, BAAS, 37, 180.09 Noeske, K. G., Weiner, B. J., Faber, S. M., et al. 2007, ApJL, 660, L43 Oke, J. B., \& Gunn, J. E. , 1983, ApJ, 266, 713

Rieke, G. H., Wright, G. S., Böker, T., et al. 2015, PASP, 127, 584 Rieke, M. J., Kelly, D., \& Horner, S. 2005, Proc. SPIE, 5904, 1 Rodighiero, G., Daddi, E., Baronchelli, I., et al. 2011, ApJL, 739, L40 Santini, P., Ferguson, H. C., Fontana, A., et al. 2015, ApJ, 801, 97 Stark, D. P., Schenker, M. A., Ellis, R., et al. 2013, ApJ, 763, 129 Tremonti, C. A., Heckman, T. M., Kauffmann, G., et al. 2004, ApJ, 613 898

Wright, G. S., Wright, D., Goodson, G. B., et al. 2015, PASP, 127, 595 Zackrisson, E., Binggeli, C., Finlator, K., et al. 2017, ApJ, 836, 78

Zackrisson, E., Rydberg, C.-E., Schaerer, D., Östlin, G., \& Tuli, M. 2011, ApJ, 740, 13 